KCL-MTH-05-09

hep-th/0507152

\title{
Arbitrary Four String Scattering at High Energy and Fixed Angle
}

\author{
Nicolas Moeller and Peter West \\ King's College London \\ Department of Mathematics \\ London WC2R 2LS, UK
}

\begin{abstract}
We calculate, using the group theoretic approach to string theory, the tree and one loop scattering of four open and closed arbitrary bosonic string states. In the limit of high energy, but fixed angle, the multi-string vertex at tree and one loop levels that we find takes a very simple form. We propose, and present arguments for, a form for the high energy multi-string vertex at all loops; in particular we give a path integral derivation of this vertex. Our results agree with those of Gross and Mende for tachyon scattering amplitudes, but those for any other string scattering are substantially different from that discussed in reference [5]. We also develop some of the technology used in the group theoretic method to compute loop corrections.
\end{abstract}




\section{Introduction}

Long ago in the early days after the discovery of string theory, high energy fixed angle scattering of the open string four tachyon scattering was considered [1]. Extremising the known one loop amplitude it was found that the non-planar process possessed a saddle point and the amplitude was evaluated at that point. This study was taken up in the late eighties [2-5]. These authors considered the closed string in the same limit and found that the amplitude for four tachyon scattering possesses a saddle point at all genus at which the positions of the Koba-Nielsen points were fixed and whose cross ratio was related to the angle of scattering. Furthermore, a concise formula for four tachyon scattering for any genus was derived in the high energy, fixed angle limit. An important role in this analysis was played by the representation of the string world surface by the Riemann sphere with two branch cuts whose degree was the genus. In reference [5] it was proposed that there existed relations between any string scattering amplitude and that for the same number of tachyon scattering that was independent of the genus. The study of the open string was also extended in reference [4].

Some interesting papers have also considered high energy scattering stressing the role of the decoupling of zero norm physical states [6].

In a theory that possesses a symmetry that is spontaneously broken, the symmetry, although present at energy scales below the breaking scale, is obscured; however, above this scale the amplitudes display the symmetry in a more transparent way. As such, it has been hoped that the study of string theory at high energy will shed some light on the underlying symmetries. In more recent times it has been proposed that $M$ theory should possess a very large Kac-Moody algebra called $E_{11}$ [7]. This was proposed by studying the maximal supergravity theories that encode all perturbative and non-perturbative effects of string theory at low energy. The motivation for this paper was to study the results of references [1-5] to see if these can make contact with this proposed large symmetry.

We will study the high energy, fixed angle, scattering of arbitrary physical bosonic string states at tree and one loop level. The most efficient method to study arbitrary string scattering is to use multi-string scattering vertices. These emerged initially from factorizing tachyon string scattering amplitudes, and was carried out in the earliest days of string theory. By sewing such vertices the pioneers of string theory were able to calculate multi-string amplitudes, so lying the foundations for string theory. In particular, they computed the one loop open string scattering amplitudes [26-32] and developed a projector which was used to eliminate the ghosts for this amplitude [33]. For two reviews which set out some of this remarkable progress, see reference [8]. These results were found before our present understanding of the systematic BRST techniques to add ghosts and so the old results often suffered from the presence of spurious ghosts. Their other problem was that the multi-string vertices they found emerged from factorising tachyon amplitudes and their properties were not well understood.

In the late eighties the study of multi-string vertices was taken up again in the socalled group theoretic approach [9-22]. The essence of this approach was that the multistring vertices were the simplest objects in string theory. It was found that multi-string vertices satisfied some very simple properties called overlap relations, and these were then formulated in such a way that they were used to define what a multi-string vertex was. 
This allowed for a systematic construction, and elaboration of the properties, of multistring vertices. For example, it allowed the incorporation of ghost oscillators, first given in an earlier paper [21], and a systematic method to change the dependence of the vertex on the Koba-Nielsen points and moduli. The amplitude was found by saturating the multistring vertex with the desired physical states which were to be scattered and integrating, with an appropriate measure, over the Koba-Nielsen points and moduli of the Riemann surface swept out by the string. As we have mentioned, the vertex is determined by certain very simple overlap relations while the measure is determined by demanding that zero norm physical states decouple. Using this technique, the scattering amplitudes for the open bosonic string [10,12-14], including ghosts $[15,16,21]$, and superstring $[10,19,20]$ were calculated. The tree level scattering for the closed string was found, but this string was not as systematically investigated as the open string. We rectify this omission in this paper. The group theoretic method was developed in the rather long series of papers [9$22]$. Although the early papers contained the essential steps, they often lacked some of the insights that were gained later on, and so they are not always so easy to read. A review was given in reference [18], but we give an account of the group theoretic method in section two of this paper in such a way that the rest of the paper can be understood in a selfcontained manner. Some aspects of the group theoretic approach, such as the integrated overlap identities, were considered in the approach of reference [23] which origined [25] in the properties of the sum over world-sheets approach to string theory.

In section three we use the group theoretic method to calculate the high energy, fixed angle, behaviour of open and closed bosonic strings scattering at tree level, while in section four we calculate four open and closed bosonic string scattering at one loop. The multistring vertices we find in the high energy limit do have an extremely simple form, that for one loop closed string scattering is given by

$$
\begin{gathered}
V_{c}=\prod_{k=1}^{N}{ }_{(k)}\langle 0| \exp \left(-\sum_{i, j} \sum_{n=1}^{\infty}\left(\alpha_{2 n}^{\mu(i)} \frac{1}{2 n\left(\varsigma_{j}-\varsigma_{i}\right)^{n}} \alpha_{0 \mu}^{(j)}+\bar{\alpha}_{2 n}^{\mu(i)} \frac{1}{2 n\left(\bar{\varsigma}_{j}-\bar{\varsigma}_{i}\right)^{n}} \alpha_{0 \mu}^{(j)}\right)\right. \\
\left.+\sum_{i, j} \alpha_{0}^{\mu(i)} \ln \sqrt{\left|\left(\varsigma_{j}-\varsigma_{i}\right)\right|} \alpha_{0 \mu}^{(j)}\right)
\end{gathered}
$$

where $\varsigma_{j}$ are the positions of the Koba-Nielsen points, which are fixed to be at the beginning and end of the two branch cuts on the Riemann sphere that represents the string worldsheet. We conjecture, with supporting evidence, the results for arbitrary genus in section seven.

In sections five and six we further develop some of the aspects of the group theoretic method associated with the calculation of loop string scattering.

Our results for four tachyon scattering in the high energy, fixed angle, limit agree with those of references [1-4] and so we agree with the majority of the work in these papers. However, we find that our results for physical states other than tachyons differ substantially from those of reference [5]. Indeed, we find that some of the amplitudes which are non-zero in reference [5] vanish in the extreme high energy limit. As a result, we do not find the 
all genus symmetry relations found in reference [5]. At the end of section four we pinpoint where we believe the problems arise in reference [5].

\section{Review of the group theoretic approach}

Here we review the group theoretic approach to string theory, which was developed in references [9-20]. The following results will be valid for both open and closed bosonic strings, and with suitable extensions the superstrings. As $N$ strings scatter they sweep out a world-sheet which is a two dimensional surface of genus $g$ with some marked points, or punctures, where the outgoing strings leave the surface. We refer to these points as Koba-Nielsen points. The string theory possesses certain conformal operators, denoted generically by the symbol $R(z)$, which depend on the world-surface, and act on the string Hilbert space $\mathcal{H}$. By definition a conformal operator of weight $d$ behaves under a conformal transformation $\xi \rightarrow \xi^{\prime}(\xi)$, where $\xi$ is a local coordinate on the world-surface, as

$$
R(\xi) \rightarrow R^{\prime}\left(\xi^{\prime}\right)=U R(\xi) U^{-1}=R(\xi)\left(\frac{d \xi}{d \xi^{\prime}}\right)^{d}
$$

Here $U$ is the operator on the string Hilbert space that corresponds to the conformal transformation $z \rightarrow z^{\prime}(z)$. The operator $U$ can be written locally in the form

$$
U=\exp \left(\sum_{n=-\infty}^{\infty} a_{n} L_{n}\right)
$$

For the open string we only consider operators of this type, but for closed strings conformal operators have left and right weights $(d, \bar{d})$, but we will mainly only require operators with a weight which is either $(d, 0)$ as above or $(0, \bar{d})$ in which case we will have the conformal transformation on the coordinate $\bar{z}$ be implemented by $\bar{L}_{n}$. For example, for the open bosonic string, the conformal operator is given by $\partial Q^{\mu}$ which has conformal weight one. Equation (2.1) also holds for the embedding field itself $Q^{\mu}$ with weight zero. The energy momentum tensor $T(z)$ transforms with weight two, but also has an anomalous term which involves the Schwarzian derivative of the conformal transformation. In particular, it obeys $T^{\prime}\left(z^{\prime}\right)=T(z)\left(\frac{d z}{d z^{\prime}}\right)^{2}+\frac{c}{12}(S z)\left(z^{\prime}\right)$ where $(S f)(z)=\frac{f^{\prime \prime \prime}(z)}{f^{\prime}(z)}-\frac{3}{2}\left(\frac{f^{\prime \prime}(z)}{f^{\prime}(z)}\right)^{2}$ and $c$ is the central charge associated with $T$. We also have the ghosts $b$ and $c$ of conformal weights two and -1 respectively.

In the group theoretic approach, the scattering amplitude, at genus $g$, of $N$ arbitrary on-shell physical states $\left|\chi_{i}\right\rangle$ is given in terms of a vertex $V$, by the expression

$$
A\left(\left|\chi_{1}\right\rangle,\left|\chi_{2}\right\rangle, \ldots,\left|\chi_{N}\right\rangle\right)=\int_{\mathcal{M}_{g, z_{k}}} \Pi_{r} d v_{r} \Pi_{i}^{\prime} d z_{i} f_{m}\left(z_{i}, v_{r}\right) V\left|\chi_{1}\right\rangle_{(1)}\left|\chi_{2}\right\rangle_{(2)} \ldots\left|\chi_{N}\right\rangle_{(N)}
$$

Here $\mathcal{M}_{g, z_{k}}$ is the moduli space of Riemann surfaces of genus $g$ with $N$ marked points $z_{i}$, i.e the Koba-Nielsen points. The integration runs over both the $z_{i}$ and over the moduli $v_{r}$. The ways of representing the two dimensional surface will be discussed later. The function 
$f_{m}\left(z_{i}, v_{r}\right)$ is the measure, and $V$ is what we call the vertex. It is a multi-linear map from $N$ copies of the Hilbert space of the string into the complex numbers:

$$
V: \mathcal{H}_{1} \otimes \ldots \otimes \mathcal{H}_{N} \rightarrow \mathbf{C}
$$

It depends on the Koba-Nielsen variables and on the moduli. The same vertex provides the scattering for any physical states once it is saturated with the corresponding physical states as in equation (2.3). Thus the vertex does not depend on what physical states are scattering, and therefore once we know the vertex and the measure, all scattering amplitudes at genus $g$ are treated on the same footing. We will now define what is meant by the vertex and show how it and the measure are determined.

The string vertex $V$ is defined by demanding that it satisfy the overlap equations

$$
V R^{(i)}\left(\xi_{i}\right)=V R^{(j)}\left(\xi_{j}\right)\left(\frac{d \xi_{j}}{d \xi_{i}}\right)^{d} .
$$

Here $\xi_{i}$ and $\xi_{j}$ denote coordinate patches on the string world-surface that are valid in the neighbourhood of the Koba-Nielsen points $z_{i}$ and $z_{j}$ respectively. The coordinate $\xi_{i}$ associated with the Koba-Nielsen point $z_{i}$ is required to vanish at this point, i.e. $\xi_{i}\left(z_{i}\right)=0$, and similarly $\xi_{j}\left(z_{j}\right)=0$. Furthermore, the two coordinates are related in their overlap region by an analytic transformation which we denote by $\xi_{j}=\tau_{j i}\left(\xi_{i}\right)$. In equation (2.5) it is important to realise that the conformal operator $R^{(j)}$ of weight $d$ acts on the $j$ th leg, or string Hilbert space of the vertex. This equation states that when the action of two conformal operators on the vertex are defined in an overlap region then each operator gives the same result when referred to the same coordinate system. Equation (2.5) holds for the conformal operators used to construct the string under consideration. For the closed bosonic string these include $x^{\mu}$ and $\partial x^{\mu}$ and the energy momentum tensor $T$ provided one includes its anomalous term. Of course, some overlap identities can be derived as a consequence of some others. The obvious example being that $\partial x^{\mu}$ follows from the identity for $x^{\mu}$ by differentiation of the overlap equation. At first sight it may appear that equation (2.5) just relates the conformal properties of an operator $R$ of weight $d$, however, the operators $R$ on the left and right hand side of the equation act on different one-string Hilbert spaces in a highly non-trivial manner.

One is free to choose whatever coordinate system one likes provided it obeys the above criterion, and one finds correspondingly different string vertices. In fact the vertex only depends on the transition functions $\tau_{j i}$ between the coordinate patches rather than the local coordinates themselves as only the former quantities actually enter into the overlap equations. To see this we write $\xi_{j}$ in equation $(2.5)$ as $\tau_{j i}\left(\xi_{i}\right)$ and we note that $\xi_{i}$ plays the role of a dummy variable. It is often useful to refer the coordinates $\xi_{j}$ to some underlying coordinate $z$, one choice of which we will discuss shortly, and then we may write

$$
\xi_{i}(z)=\sum_{n=1}^{\infty} a_{n}^{i}\left(z-z_{i}\right)^{n} \equiv\left(V_{i}\right)^{-1}(z) \quad, \quad a_{1}^{i} \neq 0 .
$$

In terms of the coordinate $z$, the Koba-Nielsen points are denoted by $z=z_{i}, i=1, \ldots, N$ and it has the above form as $\xi_{i}\left(z_{i}\right)=0$. We note that the transition functions are given 
by $\tau_{j i}=\left(V_{j}\right)^{-1} V_{i}$. The choice of any local coordinates $\xi_{i}$, subject to the above conditions, corresponds to taking any values of the constants $a_{n}$. However, as we will see different choices of local coordinates lead to the same physical scattering amplitudes.

The expression $V R^{(i)}\left(\xi_{i}\right)$ can be expressed as a Laurent series in $\xi_{i}$ around $\xi_{i}=0$, but when $\xi_{i}$ approaches another Koba-Nielsen point, say $z_{j}$, this series diverges in general. To obtain a convergent expression as one approaches $z_{j}$ one uses the overlap identity of equation (2.5) in the overlap region where both $V R^{(i)}\left(\xi_{i}\right)$ and $V R^{(j)}\left(\xi_{j}\right)$ are convergent, and then approaches the point $z_{j}$ using the latter expression. As such, the domain of validity of the coordinate $\xi_{i}$ is defined in this sense of convergent series rather than in the sense of coordinate patches as used in the theory of differentiable manifolds.

For scattering at genus zero the world-sheet is just the Riemann sphere with the marked Koba-Nielsen points and we can introduce a coordinate $z$ which covers the whole sphere with the exception of the point at infinity where we use the coordinate $\zeta=\frac{1}{z}$. If none of the Koba-Nielsen points are at infinity, one can use the local coordinates $\xi_{i}=z-z_{i}$, but there are of course an infinite number of other choices. Should it happen that one has a Koba-Nielsen point at infinity then one can use the local coordinate $\zeta$ for that string.

For surfaces of genus greater than zero the string world-surface is a Riemann surface with marked points and handles. We may use either the Schottky or Fuchsian representation of the surface. In the Fuchsian representation of the Riemann surface, every Riemann surface of genus $g \geq 2$ is conformally equivalent to the quotient of the upper-half plane by a fixed point free Fuchsian group (a discontinuous subgroup of $\operatorname{PSL}(2, R)$ ). While a genus one surface can be mapped to a parallelogram on the complex plane whose opposing sides are identified. Hence, for all genus surfaces we need only one coordinate $z$, with certain identifications

$$
z \sim P_{n} z, n=1, \ldots, 2 g
$$

where $P_{n}$ are generators of the Fuchsian group if $g \geq 2$, or in the case of genus one the two translations that identify the opposing sides of the parallelogram. When considering the open string we will double the open string world-surface to work with a more conventional Riemann surface.

We now demand that the vertex, by definition, satisfy the further overlap conditions, namely

$$
V R^{(i)}\left(\xi_{i}\right)=V R^{(i)}\left(P_{n}^{i} \xi_{i}\right)\left(\frac{d\left(P_{n}^{i} \xi_{i}\right)}{d \xi_{i}}\right)^{d}, \quad n=1, \ldots, 2 g
$$

Essentially this equation just states that $V R$ is defined on the actual surface. Using equation (2.5) to change equation (2.8) from leg $i$ to $\operatorname{leg} j$ we find that

$$
P_{n}^{j}=\tau_{j i} P_{n}^{i}\left(\tau_{j i}\right)^{-1} \quad \text { or } \quad V_{i} P_{n}^{i}\left(V_{i}\right)^{-1}=V_{j} P_{n}^{j}\left(V_{j}\right)^{-1} \equiv P_{n} .
$$

In carrying out this step we have expressed $\xi_{i}$ as $\xi_{i}=\tau_{i j} \xi_{j}$, the fact that the argument $P_{n}^{i} \xi_{i}$ of $R^{(i)}$ changes using the overlap to $R^{(j)}$ with argument $\tau_{j i}\left(P_{n}^{i} \xi_{i}\right)$ and that $\left(\tau_{j i}\right)^{-1}=\tau_{i j}$. In the last part of equation (2.9) we have used the expression for the transition functions in terms of the $V_{i}$ and as a result

$$
P_{n}^{i}=\left(V_{i}\right)^{-1} P_{n} V_{i}
$$


We recognise $P_{n}$ as the identification expressed in terms of the $z$ coordinate system as in equation (2.7). The story for the Schottky representation of the surface is similar, but one has fewer overlap conditions as one has fewer identifications. The reader is referred to reference [13] for an account.

Hence, the vertex $V$ is just defined to satisfy equation (2.5) and, in the case of loop corrections, equation (2.8). What is far from obvious, but true, is that these two equations determine the string vertex $V$ up to a function of the Koba-Nielsen coordinates $z_{i}$ and moduli $v_{r}$. One may choose this function by taking $V \prod_{i}|0\rangle_{(i)}=1$, but any other choice of the function is acceptable and will be taken into account when one computes the measure.

The amplitude of equation (2.3) also involves the measure $f$ which is determined from the requirement that the amplitude should vanish if any of the $\left|\chi_{i}\right\rangle$ is a zero norm physical state. This means that

$$
A\left(\left|\chi_{1}\right\rangle,\left|\chi_{2}\right\rangle, \ldots,\left|\chi_{N}\right\rangle\right)=0
$$

if any of the $\left|\chi_{i}\right\rangle$ are physical zero norm states. This requirement is similar to the decoupling of such states in quantum electrodynamics and so we can think of equation (2.11) as a kind of Ward identity. In fact, this fixes the measure $f_{m}$ up to a constant. The constant is determined as in the old days of sewing, or the sum over world-surfaces approach, by requiring that the scattering amplitude is unitary.

To summarize the group theoretic approach, the string vertex $V$ is by definition an object which obeys the overlap equations (2.5) and (2.8) corresponding to the conformal operators used to define the string theory, the string scattering amplitude is given by equation (2.3) and the measure is determined by demanding that zero norm physical states decouple. This is the complete system required to find the scattering of arbitrary string states.

Most of the vertex can be found more easily from the so called integrated overlap identities which we now derive from the unintegrated overlap identities above. Let us consider the expression

$$
V\left(\oint_{0} d \xi_{i} \varphi R^{(i)}\left(\xi_{i}\right)\right)
$$

where $\varphi$ is a meromorphic tensor of weight $1-d$, that is under a conformal transformation it transforms as

$$
\varphi(\xi) \rightarrow \varphi^{\prime}\left(\xi^{\prime}\right)=\varphi(\xi)\left(\frac{d \xi}{d \xi^{\prime}}\right)^{1-d}
$$

As a result, $V \varphi R^{(i)}\left(\xi_{i}\right)$ is a tensor of weight one, or simply, a one form. Let us now consider deforming the contour around $z_{i}$ such that it encloses any other non-analytic behaviour of $V \varphi R^{(i)}\left(\xi_{i}\right)$. We take an arbitrary $\varphi$ of weight $1-d$ provided it is analytic except for poles around the other Koba-Nielsen points. Thus we find a set of contour integrals around the Koba-Nielsen points. In carrying out this contour deformation one approaches the neighbourhood of other Koba-Nielsen points and, as explained above, one must use the overlap equation (2.5) to maintain convergence. Thus the overlap relations are the essential ingredient in the derivation. For the case of genus zero the contour is pulled off to infinity where it vanishes, provided this is not a Koba-Nielsen point, while at higher genus we find 
a contour around the boundary of the region which represents the Riemann surface. Since $\varphi$ is defined on the surface, that is it is suitably periodic meaning

$$
\varphi\left(\xi_{i}\right)=\varphi\left(P_{n}^{i} \xi_{i}\right)\left(\frac{d\left(P_{n}^{i} \xi_{i}\right)}{d \xi_{i}}\right)^{1-d}
$$

this contour vanishes using equation (2.8). Thus we find the integrated overlap equation

$$
\sum_{j} V\left(\oint_{0} d \xi_{j} R^{(j)}\left(\xi_{j}\right) \varphi\left(\frac{d \xi_{j}}{d \xi_{i}}\right)^{d-1}\right)=0
$$

where we have kept explicit the transformation of $\varphi$ under a change of coordinates by writing the derivative term in front of $\varphi$; this is consistent with the previous literature on the group theoretic approach. We could also have written $\sum_{j} V\left(\oint_{0} d \xi_{j} R^{(j)}\left(\xi_{j}\right) \varphi\left(\xi_{j}\right)\right)=0$, but then we have to remember that $\varphi$ is a tensor that transforms as in (2.13).

The vertex by definition satisfies the overlap equations (2.5) and (2.8), and to discover its properties it is often simpler to find how these equations behave under the desired transformation than to look into the details of the vertex itself. In particular we now consider the effect of conformal transformations on the vertex. Let us consider a conformal transformation $\xi \rightarrow \mathcal{N}(\xi)$ and denote by the same symbol $\mathcal{N}$ the operator of the realisation of the same conformal transformation on the string Fock space. The reader can distinguish from the context which $\mathcal{N}$ is required. We can carry out different conformal transformations on the different string Hilbert spaces, and as before the superscript indicates which Hilbert space the operator is acting on. For instance, $\mathcal{N}^{(i)}$ acts on the $i$ th Hilbert space. We can define a new vertex by

$$
V\left(\hat{z}_{i}, \hat{v}_{r}, \hat{\xi}_{i}\right)=V\left(z_{i}, v_{r}, \xi_{i}\right) \Pi_{k} \mathcal{N}_{k}^{(k)}
$$

The action of the conformal transformation may change the Koba-Nielsen points $z_{i}$, the moduli $v_{r}$ and the local coordinates $\xi_{i}$ used to define the vertex. We denote the new quantities with hats and show the explicit dependence of the vertex on them. The subscript on $\mathcal{N}_{k}^{(k)}$ indicates the possibility to carry out different conformal transformations on different string Hilbert spaces or string legs. In general the operator $\mathcal{N}$ has the form

$$
\mathcal{N}_{k}^{(k)}=\exp \left(\sum_{n=-\infty}^{\infty} c_{n}^{k} L_{n}^{(k)}\right)
$$

where $c_{n}^{k}$ are arbitrary constants. Under the above conformal transformation the vertex may also scale by a function of $v_{r}$ and $z_{j}$ which we have not explicitly indicated. As mentioned above, the behaviour of the vertex under a conformal transformation can be understood by studying its effect on the overlap equations. Using the properties of conformal operators of equation (2.1) we find that the overlap of equation (2.5) becomes

$$
V\left(\hat{z}_{i}, \hat{v}_{r}, \hat{\xi}_{i}\right) R^{(i)}\left(\mathcal{N}_{i}^{-1}\left(\xi_{i}\right)\right)=V\left(\hat{z}_{i}, \hat{v}_{r}, \hat{\xi}_{i}\right) R^{(j)}\left(\mathcal{N}_{j}^{-1}\left(\xi_{j}\right)\right)\left(\frac{d\left(\mathcal{N}_{j}^{-1}\left(\xi_{j}\right)\right)}{d\left(\mathcal{N}_{i}^{-1}\left(\xi_{i}\right)\right)}\right)^{d}
$$


Writing $\xi_{j}=\tau_{j i}\left(\xi_{i}\right)$ and making the change in the dummy variable $\left(\mathcal{N}_{i}^{(i)}\right)^{-1} \xi_{i}$ to $\hat{\xi}_{i}$ we find that the overlap relation becomes

$$
V\left(\hat{z}_{i}, \hat{v}_{r}, \hat{\xi}_{i}\right) R^{(i)}\left(\hat{\xi}_{i}\right)=V\left(\hat{z}_{i}, \hat{v}_{r}, \hat{\xi}_{i}\right) R^{(j)}\left(\mathcal{N}_{j}^{-1} \tau_{j i} \mathcal{N}_{i}\left(\hat{\xi}_{i}\right)\right)\left(\frac{d\left(\mathcal{N}_{j}^{-1} \tau_{j i} \mathcal{N}_{i}\left(\hat{\xi}_{i}\right)\right)}{d \hat{\xi}_{i}}\right)^{d}
$$

Interpreting the argument of the operator $R$ on the right hand side of this equation as the new local coordinate, $\hat{\xi}_{j}=\hat{\tau}_{j i}\left(\hat{\xi}_{i}\right)$, we find that the new local coordinates and Koba-Nielsen points of the vertex after the conformal transformation are given by the equation

$$
\hat{\tau}_{j i}=\left(\mathcal{N}_{j}\right)^{-1} \tau_{j i} \mathcal{N}_{i}
$$

for all $i, j=1, \ldots, N$. The Koba-Nielsen points are encoded as they are the points where $\hat{\xi}_{j}=0$ for each $j$. Carrying out a similar computation on the overlap equation (2.8), we conclude that the moduli of the vertex, which are encoded in the identifications $P_{n}^{i}$, after the conformal transformation are given by the equation

$$
\hat{P}_{n}^{i}=\left(\mathcal{N}_{i}\right)^{-1} P_{n}^{i} \mathcal{N}_{i}, i=1, \ldots, N
$$

Although one can work entirely in terms of the overlap functions, it is advantageous to work in terms of the coordinate $z$ discussed above. To this end we use $\tau_{j i}=\left(V_{j}\right)^{-1} V_{i}$ and then equation (2.20) becomes

$$
V_{j} \mathcal{N}_{j}\left(\hat{V}_{j}\right)^{-1}=V_{i} \mathcal{N}_{i}\left(\hat{V}_{i}\right)^{-1} \equiv \mathcal{N} \text { or } \mathcal{N}_{i}=\left(V_{i}\right)^{-1} \mathcal{N} \hat{V}_{i}
$$

We recognise $\mathcal{N}$ as a conformal transformation acting on the $z$ coordinate system. Using equations (2.9) and (2.22), we find that equation (2.21) can be written as

$$
\hat{P}_{n}=(\mathcal{N})^{-1} P_{n} \mathcal{N}
$$

Let us consider a conformal transformation which leaves the moduli $v_{r}$ inert, but changes the positions of the Koba-Nielsen points and the local coordinate systems $\xi_{i} \rightarrow \hat{\xi}_{i}=$ $\left(\hat{V}_{i}\right)^{-1} z$ around each Koba-Nielsen point. From equation (2.23) such a transformation will have $P_{n}=\hat{P}_{n}$ and so we may take $\mathcal{N}=I$ and, from equation $(2.22)$, have $\mathcal{N}_{i}=\left(V_{i}\right)^{-1} \hat{V}_{i}$. Acting on the vertex we have

$$
V\left(v_{r}, \hat{z}_{j}, \hat{\xi}_{j}\right)=V\left(v_{r}, z_{j}, \xi_{j}\right) \prod_{i=1}^{N}\left(V_{i}\right)^{-1} \hat{V}_{i}
$$

The new Koba-Nielsen points are given by the points where $\hat{\xi}_{j}$ vanish for each $j$. This is $\hat{\xi}_{j}\left(\hat{z}_{j}\right)=0$, or equivalently $\left(\hat{V}_{j}\right)^{-1}\left(\hat{z}_{j}\right)=0$. Transformations $\mathcal{N}_{i}$ of this kind are all analytic transformations. Those for which $\mathcal{N}_{i}(0)=0$ do not change the positions of the Koba-Nielsen points and just define new local coordinates around each Koba-Nielsen point. These are of the form of (2.17) with the sum for $n \geq 0$. Those that also shift the 
Koba-Nielsen points also include $L_{-1}$ in the sum. Since physical states are annihilated by $L_{n}-\delta_{n, 0}, n \geq 0$ the former transformations do not affect physical amplitudes except for a scaling due to the $L_{0}$ term. However, the $L_{0}$ term may scale the vertex by a factor, but this is compensated for when the measure is calculated. The same conclusion applies to the transformations that shift the Koba-Nielsen points.

Now we consider an infinitesimal conformal transformation which does not change $z_{j}$, or the local coordinates, but changes the moduli $v_{r} \rightarrow \hat{v}_{r}=v_{r}+\epsilon h_{r}(v)$. In this case, $V_{j}=\hat{V}_{j}$, and we are left with $\mathcal{N}$. Then equation (2.23) applied to the coordinate $z$ becomes

$$
P_{n}(z)+\epsilon f(z) \frac{d P_{n}(z)}{d z}=\hat{P}_{n}(z)+\epsilon f\left(P_{n} z\right)
$$

where

$$
\mathcal{N}(z)=z+\epsilon f(z) .
$$

An infinitesimal conformal transformation $z \rightarrow z+\epsilon f(z)$ is realised on the string Hilbert space by the operator $I+\epsilon \oint_{0} T(z) f(z)$. Consequently, implementing the conformal transformation of equation (2.26) on the vertex we find that equation (2.16) is given by

$$
\sum_{r=1}^{g} h_{r}(v) \frac{\partial V}{\partial v_{r}}=V\left(\sum_{j=1}^{N} \oint_{\xi_{j}=0} d \xi_{j} T^{(j)}\left(\xi_{j}\right) f^{j}\left(\xi_{j}\right)+c_{f}\right)
$$

where $c_{f}$ is a function of the moduli and Koba-Nielsen points and $\xi_{i}+\epsilon f^{i}\left(\xi_{i}\right)=\mathcal{N}_{i}\left(\xi_{i}\right)=$ $\left(V_{i}^{-1} \mathcal{N} V_{i}\right)\left(\xi_{i}\right)$. The constant $c_{f}$ is required to take account of the presence of a multiplier which has not been explicitly written in equation (2.16) and the fact that the vertex is only determined up to a function of the moduli and Koba-Nielsen points by the overlap relations. The value of $c_{f}$ is easily determined by taking a suitable state, such as the vacuum state, on either side of this equation. We recall, however, that to find the actual scattering amplitude, we multiply the vertex by the measure $f_{m}$ and integrate over all the Koba-Nielsen variables and moduli. The final result is independent of what choice of multiplicative factor in front of $V$ one takes.

It is instructive to consider conformal transformations that do not change the vertex, that is they change neither $v_{r}, z_{j}$, nor the local coordinate system. When we have loops this means that $P_{n}=\hat{P}_{n}$ and so we require

$$
P_{n}^{-1} \mathcal{N} P_{n}=\mathcal{N} .
$$

If there are no loops then there are no identifications $P_{n}$ and so $\mathcal{N}$ is unrestricted. The conformal transformations on each leg are given by equation (2.22) with $V_{i}=\hat{V}_{i}$ and so

$$
\mathcal{N}_{i}=\left(V_{i}\right)^{-1} \mathcal{N} V_{i}
$$

For infinitesimal transformations, we take equation (2.25), with $P_{n}=\hat{P}_{n}$, which means that $f(z)$ transforms as a vector field (i.e., a tensor of rank -1 ) and is defined on the Riemann surface itself. Thus we find an equation of the form

$$
V\left\{\sum_{j=1}^{N} \oint_{\xi_{j}=0} d \xi_{j} \varphi\left(T^{j}\left(\xi_{j}\right) \frac{d \xi_{j}}{d \xi_{i}}+\frac{c}{12}\left(S \xi_{j}\right)\left(\xi_{i}\right) \frac{d \xi_{i}}{d \xi_{j}}\right)\right\}=0
$$


This is just an example of the overlap equation (2.5) found by taking $R(z)=L(z)$ which has conformal weight 2 and we have recognised the constant to be the Schwarzian derivative. The set of such transformations which preserve a $g$ loop vertex with $N$ external lines form a group which is discussed in reference [17].

Under the transformations

$$
V_{j} \rightarrow S V_{j}, \quad P_{n} \rightarrow S P_{n} S^{-1}
$$

the vertex is inert as it only depends on $P_{n}^{i}$ of equation (2.10) and the transition functions $\tau_{j i}=\left(V_{j}\right)^{-1}\left(V_{i}\right)$ and since these are inert under the above transformations the result follows. Indeed, the transformation properties of the vertex can be summarized by writing the dependence of the vertex $V$ on $v_{r}$ and $z_{j}, \xi_{j}$ through $P_{n}$ and $V_{j}$, i.e. $V\left(P_{n}, V_{j}\right)$ and the $\mathrm{SU}(1,1)$ invariance becomes $V\left(S P_{n} S^{-1}, S V_{j}\right)=V\left(P_{n}, V_{j}\right)$. This invariance is a feature of the redundancy of the representation in terms of $P_{n}$ and $V_{j}$ that we have used. The conformal transformation property of the vertex embodied in equations (2.22) and (2.23) may be written as

$$
V\left(P_{n}, V_{j}\right) \prod_{i=1}^{N} \mathcal{N}_{i}^{(i)}=V\left(\mathcal{N}^{-1} P_{n} \mathcal{N}, \hat{V}_{j}\right) .
$$

We have again not explicitly written the possible scaling of $V$ by a function of $v_{r}$ and $z_{j}$.

As stated above, the measure is determined by ensuring that zero-norm physical states decouple. For the open bosonic string these states are of the form

$$
L_{-1}|\Omega\rangle \quad, \quad\left(L_{-2}+\frac{3}{2} L_{-1}^{2}\right)\left|\Omega^{\prime}\right\rangle
$$

where

$$
L_{n}|\Omega\rangle=L_{n}\left|\Omega^{\prime}\right\rangle=0 \quad n>0, \quad L_{0}|\Omega\rangle=\left(L_{0}+1\right)\left|\Omega^{\prime}\right\rangle=0
$$

together with higher level zero norm states. The states for other strings have a similar form; they consist of operators which generate the symmetry algebra of the string in question acting on a state which is highest weight with respect to the algebra. Saturating the vertex with one of these states we may interpret it as an infinitesimal conformal transformation applied to the vertex times $\left|\Omega^{\prime}\right\rangle$ or $|\Omega\rangle$. Such transformations will either preserve the vertex, or change its Koba-Nielsen coordinates or moduli. Changes of local coordinate systems are irrelevant as the vertex is saturated with physical states. Consequently, we find, generically, that

$$
\int \prod_{k}^{\prime} d z_{k} \prod_{r} d v_{r} f_{m}\left(\sum_{r} h_{r} \frac{\partial V}{\partial v_{r}}+\sum_{j} g_{j} \frac{\partial V}{\partial z_{j}}-c V\right)\left|\chi_{1}\right\rangle_{(1)} \ldots|\Omega\rangle_{(i)} \ldots\left|\chi_{N}\right\rangle_{(N)}=0
$$

where all the states $|\chi\rangle_{(i)}$ are physical and so obey $\left(L_{n}-\delta_{n, 0}\right)|\chi\rangle=0, n \geq 0$ and

$$
v_{r} \rightarrow v_{r}+h_{r}\left(v_{r}, z_{j}\right), \quad z_{j} \rightarrow z_{j}+g_{j}\left(z_{j}, v_{r}\right)
$$


are the changes that the infinitesimal conformal transformation induces on the vertex. We find, by integrating by parts, that

$$
0=\sum_{r} \frac{\partial}{\partial v_{r}}\left(h_{r} f_{m}\right)+\sum_{i} \frac{\partial}{\partial z_{i}}\left(g_{i} f_{m}\right)+c f_{m}
$$

in other words a first-order differential equation for the measure, $f_{m}$. In fact, one has as many differential equations as one has Koba-Nielsen variables and moduli and so the measure is determined by them. In this calculation we have neglected boundary terms which in general are not zero and correspond to a B.R.S.T anomaly. Indeed, all open strings, except for the gauge group $\mathrm{SO}\left(2^{13}\right)$, have such an anomaly [12,25].

The process of decoupling without ghosts becomes, at least at first sight, more and more difficult to implement at higher loops due to the complicated structure of the zeronorm physical states. This difficulty is not present when one incorporates ghosts as these states are of the form $Q|\Lambda\rangle$. However, the correct vertex to use is no longer $V$, when ghost extended, as this is annihilated by $Q$ and so does not lead to any constraints on the measure. In fact, one must multiply the vertex by a ghost oscillator prefactor. This is done explicitly in references [15] and [16] and one finds that the measure obeys the differential equations

$$
\frac{\partial \ln f_{m}}{\partial z^{j}}=0=\frac{\partial \ln f_{m}}{\partial v_{r}}+c_{r}
$$

where $c_{r}$ are the constants in equation (2.27). One can, of course, redefine one's vertex by multiplying it by $f_{m}$ and then $c_{r}$ is zero and the corresponding measure is one.

This completes our review of the group theoretic method to computing string amplitudes. To illustrate how to apply the method, we compute open bosonic string scattering at the tree level. We adopt a particularly simple set of local coordinates

$$
\xi_{i}(z)=\left(V_{i}\right)^{-1}(z)=z-z_{i}, \text { or } \xi_{j}=\xi_{i}+z_{i}-z_{j}
$$

We begin by deriving the $N$ string vertex $V$. The overlap equation (2.5) for the conformal operator

$$
Q^{\mu}(z)=-\frac{\overleftarrow{\partial}}{\partial \alpha_{0}^{\mu}}-\alpha_{0}^{\mu} \ln z+\sum_{\substack{n=-\infty \\ n \neq 0}}^{\infty} \frac{\alpha_{n}^{\mu} z^{-n}}{n}
$$

of conformal weight zero is given by

$$
V Q^{\mu(i)}\left(z-z_{i}\right)=V Q^{\mu(j)}\left(z-z_{j}\right)
$$

For $R(z)=P^{\mu}(z) \equiv \partial Q^{\mu}(z)$, the integrated overlap equation (2.15) becomes

$$
V\left(\sum_{j=1}^{N} \oint d \xi_{j} \varphi_{n} P^{\mu(j)}\left(\xi_{j}\right)\right)=0
$$


We can take $\varphi_{n}$ to be of the form

$$
\varphi_{n}=\frac{1}{\left(\xi_{i}\right)^{n}}=\frac{1}{\left(\xi_{j}+z_{j}-z_{i}\right)^{n}}=\sum_{p=0}^{\infty}\left(\begin{array}{c}
-n \\
p
\end{array}\right) \frac{\left(\xi_{j}\right)^{p}}{\left(z_{j}-z_{i}\right)^{n+p}} \quad \forall j \neq i
$$

In fact, this set of $\varphi$ 's form a complete set of all allowed $\varphi$ 's. Substituting the $\varphi$ 's into equation (2.42) and using the relation

$$
P^{\mu}(z)=\sum_{n=-\infty}^{\infty} \alpha_{-n}^{\mu} z^{n-1}
$$

we find the identity

$$
0=V\left(\alpha_{-n}^{\mu(i)}+\sum_{\substack{j=1 \\
j \neq i}}^{N} \sum_{p=0}^{\infty}\left(\begin{array}{c}
-n \\
p
\end{array}\right) \frac{\alpha_{p}^{\mu(j)}}{\left(z_{j}-z_{i}\right)^{n+p}}\right)
$$

The solution to this equation gives us the vertex for the open string

$$
\begin{aligned}
V=\prod_{k=1}^{N}{ }_{(k)}\langle 0| \exp & \left(\sum _ { \substack { i , j \\
i \neq j } } \left\{-\frac{1}{2} \sum_{n, m=1}^{\infty} \alpha_{n}^{\mu(i)} \frac{(n+m-1) !(-1)^{m}}{m ! n !\left(z_{j}-z_{i}\right)^{n+m}} \alpha_{m \mu}^{(j)}\right.\right. \\
& \left.\left.-\sum_{n=1}^{\infty} \frac{\alpha_{n}^{\mu(i)} \alpha_{0 \mu}^{(j)}}{n\left(z_{j}-z_{i}\right)^{n}}-\frac{1}{2} \alpha_{0}^{\mu(i)} N^{i j} \alpha_{0 \mu}^{(j)}\right\}\right)
\end{aligned}
$$

where $N^{i j}$ is not determined by the integrated overlap and we have arbitrarily chosen the normalization of $V$. To find $N^{i j}$, one uses the unintegrated $Q^{\mu}$ overlap of equation (2.41) and one finds the result to be

$$
N^{i j}=-\ln \left|\left(z_{j}-z_{i}\right)\right|
$$

One could, from the beginning, have determined $V$ completely from the $Q^{\mu}$ overlap, however, it is technically simpler to first carry out the partial determination given above with the integrated $P^{\mu}$ overlap. It is straightforward to evaluate the vertex for any choice of $\left(V_{i}\right)^{-1}$, or local coordinates.

We have used a somewhat short hand notation in equation $(2.46)$. By $\prod_{k=1(k)}^{N}\langle 0|$ we actually mean

$$
\prod_{k=1}^{N} \int d^{D} q_{k} \delta\left(\sum_{l} q_{l}^{\mu}\right){ }_{(k)}\left\langle 0, q_{k}\right|
$$

where $|0, p\rangle$ obeys $\alpha_{n}^{\mu}|0, p\rangle=0, n \geq 1$ and $p^{\mu}$ is its momentum. 
We find that a small change, $z_{j} \rightarrow \hat{z}_{j}=z_{j}+\epsilon, z_{i} \rightarrow \hat{z}_{i}=z_{i}$ for $i \neq j$ implies that $\left(V_{j}\right)^{-1} \hat{V}_{j}(z)=z+\hat{z}_{j}-z_{j}=z+\epsilon$ and $\left(V_{i}\right)^{-1} \hat{V}_{i}(z)=z$ for $i \neq j$. The induced transformation has $\mathcal{N}_{j}=\exp \left(\epsilon L_{-1}^{(j)}\right)$ all conformal transformations on the other string Hilbert spaces being the identity operator. As a result equation (2.24) implies that

$$
\frac{\partial V}{\partial z_{j}}=V L_{-1}^{(j)}
$$

We can now determine the measure. Applying the zero norm physical state $L_{-1}^{(j)}|\Omega\rangle_{j}$ on the $j$ th string Hilbert space and arbitrary physical states $\left|\chi_{k}\right\rangle$ to all the other Hilbert spaces to the integrated vertex

$$
W=\int \prod_{j=1}^{N-3} d z_{j} f_{m} V
$$

we find that

$$
W|\chi\rangle_{(1)} \ldots|\chi\rangle_{(N)}=\int \prod_{k=1}^{N-3} d z_{k} f_{m} \frac{\partial V}{\partial z_{j}}|\chi\rangle_{(1)} \ldots|\Omega\rangle_{(j)} \ldots|\chi\rangle_{(N)} \quad ; \quad j=1, \ldots, N-3
$$

which implies that $\frac{\partial f_{m}}{\partial z_{j}}=0$, or that $f_{m}$ is a constant. One must also show that the state $L_{-1}^{(j)}|\Omega\rangle_{(j)}$ decouples for $j=N-2, N-1, N$. This is achieved by using the three vertex preserving integrated overlaps of equation (2.30), which follow from taking $\varphi$ to be the three analytic vector fields that live on the Riemann sphere, namely $\varphi=1, z, z^{2}$. This is related to the $\mathrm{SU}(1,1)$ invariance of the result which is discussed using these identities in reference [14]. To complete the discussion of decoupling, one must also show that states of the form $\left(L_{-2}+\frac{3}{2} L_{-1}^{2}\right)\left|\Omega^{\prime}\right\rangle$ decouple. This is shown using the vertex preserving integrated overlaps containing $L_{-2}^{j}$ on one leg.

Thus we have completely determined the open string tree level vertex $V$ and the measure $f_{m}$. To find the scattering amplitude for any desired physical states, one simply saturates $W$ with them.

The derivation of the vertex for genus greater than one follows a similar path. However, one must consider integrated overlap identities for functions that are allowed to shift by constants under the periodicity relations of the surface. In this case one also finds contributions coming from the boundaries that represent the Riemann surface. This allows one to take account of the fact that strictly periodic scalar functions having only a single pole at a given point do not exist if the pole is of certain degrees.

We note that in the older literature on the group theoretic approach (except the more recent [22]), the overlap equations look a bit different because a different convention for a conformal operator was used. In this paper we use the more common definition given in equation (2.1). while in the previous literature, $\left(\xi_{i}\right)^{-d}$ was factored out of the operator, which led to the transformation rule

$$
\frac{R\left(\xi_{i}\right)}{\left(\xi_{i}\right)^{d}} \rightarrow \frac{R\left(\xi_{j}\right)}{\left(\xi_{j}\right)^{d}}\left(\frac{d \xi_{j}}{d \xi_{i}}\right)^{d}
$$


The same group theoretic approach can be applied to superstrings provided one makes the appropriate generalisations. We refer the reader to references $[10,19,20]$ for such discussion.

Although the group theoretic approach has so far only been used to construct the scatterings in string theories that are based on free field constructions, it could be used for string theories in general and in particular those based on $N=2$ minimal models. The latter are associated with dimensional reductions on Calabi-Yau spaces. The conformal operators are the primary fields that occur in the minimal models and lead to corresponding overlap equations. The measure is then determined by the decoupling of zero norm physical states.

\section{High Energy Tree Level Arbitrary String Scattering}

We consider the limit in which $\alpha^{\prime} \rightarrow \infty$, but the angle of scattering is fixed. In this section we find the behaviour of open bosonic strings at the tree level. In this limit $\alpha_{0} \rightarrow \infty$ and $V \alpha_{0}^{\mu(i)} \gg V \alpha_{n}^{\mu(i)}$ for $n$ a positive integer, and so we neglect terms of the latter form. However, we must keep terms of the form $V \alpha_{-n}^{\mu(i)}$ for $n$ a positive integer as these may lead to $V \alpha_{0}^{\mu(j)}$ using the overlap relations. We could just extract the high energy vertex from equation (2.46), but it is more instructive to derive the vertex from first principles in the high energy limit.

We adopt the simple local coordinate systems of equation (2.39). Taking $R=\partial x^{\mu}$, the overlap equation (2.15), for the functions of equation (2.43), and in the high energy limit reads

$$
V\left(\alpha_{-n}^{\mu(i)}+\sum_{j, j \neq i} \frac{\alpha_{0}^{\mu(j)}}{\left(z_{j}-z_{i}\right)^{n}}\right)=0
$$

As such, the vertex is of the form

$$
V=\prod_{k=1}^{N}{ }_{(k)}\langle 0| \exp \left(-\sum_{i \neq j} \sum_{n=1}^{\infty} \alpha_{n}^{\mu(i)} \frac{1}{n\left(z_{j}-z_{i}\right)^{n}} \alpha_{0 \mu}^{(j)}+\frac{1}{2} \sum_{i \neq j} \alpha_{0}^{\mu(i)} \ln \left|\left(z_{j}-z_{i}\right)\right| \alpha_{0 \mu}^{(j)}\right)
$$

The last term does not follow from the integrated overlap equation (3.1), but is derived from the unintegrated overlap involving $Q^{\mu}$. We note that in the high energy limit we have neglected the term involving $\alpha_{n}^{(i)} \cdot \alpha_{m}^{(j)}$ in the general vertex of equation (2.45). Naively, one may be tempted to also drop the first term in the vertex in equation (3.2) with respect to the second term, however, this is required to give the leading terms in the scattering of strings which are not tachyons.

We now consider the extremisation of the vertex with respect to the positions of the Koba-Nielsen points $z_{i}$. Equation (2.49) is valid at any energy and the vertex will be an extremum if $\frac{\partial V}{\partial z_{i}}=0$ and, as a result,

$$
V L_{-1}^{(i)}=0
$$


However, in the high energy limit $L_{-1}^{(i)}=\alpha_{-1}^{(i)} \cdot \alpha_{0}^{(i)}$, and so we conclude that

$$
0=V \alpha_{-1}^{(i)} \cdot \alpha_{0}^{(i)}=-V \sum_{j, j \neq i} \frac{\alpha_{0}^{(i)} \cdot \alpha_{0}^{(j)}}{\left(z_{j}-z_{i}\right)}
$$

where to find the last line we have used equation (3.1). Hence we conclude that the scattering at high energy is dominated by momenta that satisfy the condition

$$
\sum_{j, j \neq i} \frac{p_{i} \cdot p_{j}}{\left(z_{j}-z_{i}\right)}=0
$$

Let us examine the consequence of this relations for four particle scattering. Taking $i=1$ equation $(3.5)$ reads

$$
\frac{p_{1} \cdot p_{2}}{\left(z_{1}-z_{2}\right)}+\frac{p_{1} \cdot p_{3}}{\left(z_{1}-z_{3}\right)}+\frac{p_{1} \cdot p_{4}}{\left(z_{1}-z_{4}\right)}=0
$$

Using momentum conservation, i.e. $p_{4}=-\left(p_{1}+p_{2}+p_{3}\right)$, the fact that in the high energy limit $p_{i} \cdot p_{i}=0$ and the usual variables

$$
s=-\left(p_{1}+p_{2}\right)^{2}, \quad t=-\left(p_{1}+p_{3}\right)^{2}, \quad u=-\left(p_{1}+p_{4}\right)^{2},
$$

equation (3.6) becomes

$$
\frac{s}{t}=\frac{p_{1} \cdot p_{2}}{p_{1} \cdot p_{3}}=-\frac{\left(z_{1}-z_{2}\right)\left(z_{3}-z_{4}\right)}{\left(z_{1}-z_{3}\right)\left(z_{2}-z_{4}\right)}
$$

where on the right-hand side we recognise the cross ratio. Taking other values of $i$ we recover the same equation, once we use the relations between the various cross ratios. Thus we recover the result of references $[2,3]$ found in the context of tachyon scattering.

Any physical state is of the form

$$
\alpha_{-n_{1}}^{\mu_{1}} \ldots \alpha_{-n_{p}}^{\mu_{p}}|0, p\rangle
$$

where $|0, p\rangle$ satisfies $\alpha_{n}^{\mu}|0, p\rangle=0, n \geq 1$ and $\alpha_{0}^{\mu}|0, p\rangle=\sqrt{2 \alpha^{\prime}} p^{\mu}|0, p\rangle$. Acting with the vertex on this state, positioned in the $i$ th string Hilbert space, we may use the overlap relation of equation (3.1) to swop any $\alpha_{-n}^{\mu(i)}$ for $-\sum_{j, j \neq i} \frac{\alpha_{0}^{\mu(j)}}{\left(z_{j}-z_{i}\right)^{n}}$. Once all the oscillators have been traded in this way one is left with an expression which is polynomial in momenta times the tachyon amplitude.

For example the scattering of four photons in the high energy limit becomes

$$
V \prod_{k} \epsilon^{\mu(k)} \alpha_{-1 \mu}^{(k)}\left|0, p_{k}\right\rangle_{(k)}=\prod_{i} \sum_{j, j \neq i} \frac{1}{\left(z_{j}-z_{i}\right)} \epsilon^{\mu(i)} \alpha_{0 \mu}^{(j)}\left(V \prod_{k}\left|0, p_{k}\right\rangle_{(k)}\right)
$$


where in the expression in brackets at the end of this equation we recognise the tachyon amplitude.

These are the symmetry relations found in reference [5] at high energy. They are a consequence of using the overlap relations of the group theoretic approach involving $R=$ $\partial x^{\mu}$ to rewrite the physical states acting on the vertex. However, the overlap relations are valid at all energies and resulting identities can be derived relating all scattering amplitudes to those of the tachyons. At high energy the identities simplify considerably.

The closed string proceeds along very similar lines. Equation (3.1) holds with an analogous equation for $\bar{\alpha}_{n}^{\mu}$. The vertex is given by equation (3.2) with the addition of very similar terms for the barred oscillators which involve $\bar{z}_{j}$ 's. Equations (3.3) to (3.8) carry over unchanged. We also conclude that all amplitudes can be expressed in terms of the tachyon amplitude using the overlap relations, which are the corner stone of the group theoretic approach, at any energy, but these simplify considerably at high energy.

\section{High Energy Genus One Arbitrary String Scattering}

In this section we will find the one loop amplitudes for arbitrary open and closed string scattering in the limit of high energy, i.e. $\alpha^{\prime} \rightarrow \infty$, but with the angle of scattering fixed. As at the tree level, in this limit $\alpha_{0} \rightarrow \infty$ and $V \alpha_{0}^{\mu(i)} \gg V \alpha_{n}^{\mu(i)}$ for $n$ a positive integer, and so we neglect terms of the latter form. However, we must keep terms of the form $V \alpha_{-n}^{\mu(i)}$ for $n$ a positive integer as these may lead to $V \alpha_{0}^{\mu(j)}$ using the overlap relations. We will carry out the calculation using two different representations of the genus one surface. In the first sub-section we use the Fuchsian representation involving two identifications of the opposing sides of a parallelogram and in the next sub-section we represent the genus one surface by the Riemann sphere with two square root branch cuts. We also discuss the relationship between the results found in the two representations and explain how the differences with the results of reference [5], which used the latter representation, arise.

\subsection{Genus One Scattering using the Fuchsian Representation}

We could just extract the high energy vertex from the general one loop vertex calculated in section six, but it is more instructive to derive the vertex from first principles in the high energy limit. As such, this section is largely self contained.

At genus one the string sweeps out a surface that is a torus with marked points. A torus can be thought of as the complex plane $\mathbf{C}$ subject to the equivalence relation $z \sim z+2 n w+2 m w^{\prime}$ for $n, m \in \mathbf{Z}$ for all points $z \in \mathbf{C}$. The torus is then represented by a parallelogram whose corners we may choose to be at the points $0,2 w, 2 w+2 w^{\prime}$ and $2 w^{\prime}$ as one moves around the parallelogram in an anti-clockwise movement. For the closed string we choose $2 w=1$, while for the doubled surface for the open string we write $2 w=-\ln u$, and take $w^{\prime}=i \pi$. The torus is formed by identifying its opposing sides of the parallelogram, which is enforced by the equivalence relation. Thus the torus has the periods $2 w$ and $2 w^{\prime}$ with modulus parameter defined to be $\tau \equiv \frac{w^{\prime}}{w}$. The region inside the parallelogram is defined to be the fundamental domain and its points are in one to one correspondence with those of the genus one surface. We use the conventions of Bateman [24] and will refer to this representation of the genus one surface as the Fuchsian representation. We will 
adopt the simple local coordinates $\xi_{i}=z-z_{i}$, of equation (2.39), in the neighbourhood of the Koba-Nielsen points $z_{i}$.

We begin by considering the scattering of four closed strings. We now consider the overlap equations (2.5) involving the conformal field $\partial x^{\mu}$. Using these and the overlap relations

$$
V \partial x^{\mu}(z)=V \partial x^{\mu}(z+2 w), \quad V \partial x^{\mu}(z)=V \partial x^{\mu}\left(z+2 w^{\prime}\right),
$$

which are those of equation (2.8) for the conformal operator $\partial x^{\mu}$, we can derive the integrated overlap equation (2.15), namely

$$
\sum_{j} \oint d \xi_{j} V \varphi \partial x^{\mu(j)}\left(\xi_{j}\right)=0
$$

where $\varphi$ is a scalar which satisfies the periodicity conditions

$$
\varphi(z)=\varphi(z+2 w), \quad \varphi(z)=\varphi\left(z+2 w^{\prime}\right)
$$

and is analytic except for possible poles at the Koba-Nielsen points. The derivation is as explained in section two, the cancellation of contributions from opposite sides of the parallelogram being obvious as $\varphi$ and $V R$ are periodic.

To derive the vertex it is desirable to have a periodic function with a pole at only one Koba-Nielsen point. The Weierstrass function $\mathcal{P}(z)$ is the unique function which has a pole at order two of the form $\frac{1}{z^{2}}$ at $z=0$, and points related by the periods of the torus, but is otherwise analytic. Its derivatives $\mathcal{P}^{(m)}(z) \equiv \frac{d^{m} \mathcal{P}(z)}{d z^{m}}$ have poles of all higher orders. There is no such function with a pole of order one. This is obvious, integrating such a possible periodic function around the boundary of the parallelogram and using equation (4.1.3) we find zero. However, this is also the residue to the pole which must therefore vanish. We will discuss below how to deal with this case. Taking $\varphi=\frac{(-1)^{m}}{(m+2) !} \mathcal{P}^{(m)}\left(z-z_{i}\right)$, the overlap equation (4.1.2), in the high energy limit, reads for $n \geq 2$

$V\left(\frac{1}{n} \alpha_{-n}^{\mu(i)}+\left.\frac{(-1)^{n}}{n !} \frac{d^{n-2}}{d t^{n-2}}\left(\mathcal{P}(t)-t^{-2}\right)\right|_{t=0} \alpha_{0}^{\mu(i)}+\sum_{j, j \neq i} \frac{(-1)^{n}}{n !} \mathcal{P}^{(n-2)}\left(z_{j}-z_{i}\right) \alpha_{0}^{\mu(j)}\right)=0$

Note that in the high energy limit we keep only negative moded oscillators and the momenta acting on the vertex. As such, the only information required from $\varphi$ is the degree of the pole at the one Koba-Nielsen point and its values at the other Koba-Nielsen points.

We now restrict our attention to the scattering of four closed strings. It has been shown $[2,3]$ that the genus one closed string amplitude at high energy is dominated when the Koba-Nielsen points sit at the half periods of the torus. We now consider only such scattering, but we will show below that the amplitude is extremised in this configuration. We therefore take the Koba-Nielsen points to be at

$$
z_{1}=w, z_{2}=w+w^{\prime}, z_{3}=w^{\prime}, z_{4}=0
$$


Since the Koba-Nielsen points are on the edges of the fundamental regions we should specify how we draw the integration contour. We will adopt our contour of integration used in the integrated overlap equations to start at $-w^{\prime}$, not including this point inside the contour as it goes anti-clockwise around, move to the point $-w^{\prime}+2 w$ not including this point, then move to $w^{\prime}+2 w$ not including this point, then to $w^{\prime}$ including this point and back to the beginning. As we do so the contour is deformed so that the Koba Nielsen points within the contour are at $0, w, w+w^{\prime}$ and $w^{\prime}$ with all their images excluded from the contour. We have drawn this in figure 1.

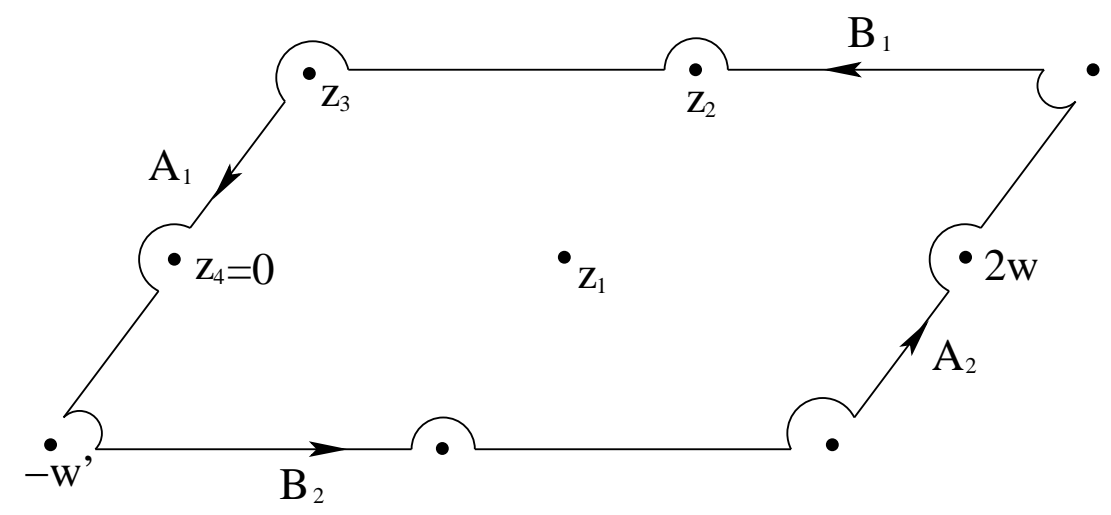

Figure 1: The contour

Now it is known [24] that $\mathcal{P}^{(2 m+1)}$, for integer $m=1,2,3, \ldots$, is equal to $\mathcal{P}^{(1)}$ times a polynomial of degree $m$ in $\mathcal{P}$. However, $\mathcal{P}^{(1)}$ vanishes at the half periods, $\mathcal{P}^{(1)}\left(z_{i}-z_{j}\right)=0$ and so we find that

$$
V \alpha_{-(2 m+1)}^{\mu(i)}=0, \quad m=1,2,3, \ldots
$$

We note that $\mathcal{P}^{(2 m-2)}$, for positive integer $m$, is equal to a polynomial of degree $m$ in $\mathcal{P}$ which does not in general vanish at the half periods.

As we noted above, there does not exist a periodic function that has only a single pole of order one. The way around this difficulty is to consider functions which are not periodic, but shift by constants under the change of periods, namely a function such that

$$
\varphi(z+2 w)=\varphi(z)+c_{1}, \quad \varphi\left(z+2 w^{\prime}\right)=\varphi(z)+c_{2}
$$

We now consider the contour integral of $V \partial x^{\mu} \varphi$ around the boundary of the torus. The contributions from opposing sides no longer cancel and one finds instead of equation (4.1.2) that

$$
\sum_{j} \oint d \xi_{j} V \varphi \partial x^{\mu(j)}\left(\xi_{j}\right)=c_{2} \int_{B_{1}} V \partial x^{\mu} d z+c_{1} \int_{A_{2}} V \partial x^{\mu} d z
$$

On the right hand side $V \partial x^{\mu} d z$ stands for $V \partial x^{\mu(k)}\left(\xi_{k}\right) d \xi_{k}$ which is independent of which string Hilbert space one uses by virtue of equation (2.5) using the operator $\partial x^{\mu}$. The vertex should not depend on which function $\varphi$ one takes as long as it obeys equation (4.1.7). Functions of this type, with a pole of order one at the same place, differ by a 
constant times $z$. Taking $\varphi=z$, which of course is a function which shifts by periods of the torus, in equation (4.1.8) we find that provided

$$
w^{\prime} \int_{B_{1}} V \partial x^{\mu} d z+w \int_{A_{2}} V \partial x^{\mu} d z=\frac{1}{2} V \sum_{j} z_{j} \alpha_{0}^{\mu(j)}
$$

we will indeed find the same result no matter what function $\varphi$ we take. In this last equation we have no $\alpha_{1}^{\mu}$ terms as we are in the high energy limit. As we will show in section six, modular invariance allows one to conclude that the solution to equation (4.1.9) is given by

$$
\int_{B_{1}} V \partial x^{\mu} d z=\frac{1}{2} V\left(\alpha_{0}^{\mu(2)}+\alpha_{0}^{\mu(3)}\right)
$$

The function

$$
\varphi_{1}(z)=\zeta(z)-\frac{\eta}{w} z
$$

obeys equation (4.1.7) with $c_{1}=0$ and $c_{2}=-\frac{i \pi}{w}$. In this equation $\zeta(z)$ is the zeta function which is defined to obey $-\frac{d \zeta(z)}{d z}=\mathcal{P}(z)$ and has a first order pole with residue one. It is not a periodic function, but obeys

$$
\zeta(z+2 w)=\zeta(z)+2 \eta, \quad \zeta\left(z+2 w^{\prime}\right)=\zeta(z)+2 \eta^{\prime}
$$

where $\eta$ and $\eta^{\prime}$ are independent of $z$. Substituting $\varphi_{1}$ into equation (4.1.8) and using equation (4.1.10) we find that

$$
V\left(\alpha_{-1}^{\mu(i)}+\sum_{j, j \neq i} \alpha_{0}^{\mu(j)} \varphi_{1}\left(z_{j}-z_{i}\right)\right)=-\frac{i \pi}{2 w} V\left(\alpha_{0}^{\mu(2)}+\alpha_{0}^{\mu(3)}\right)
$$

Evaluating $\varphi_{1}\left(z_{j}-z_{i}\right)$ we find that this equation becomes

$$
V \alpha_{-1}^{\mu(i)}=0
$$

which, together with equation (4.1.6), implies that all odd oscillators acting on the vertex vanish. The story for the overlap equations involving $\bar{\alpha}_{n}^{\mu}$ is very similar.

Using equations (4.1.4), (4.1.6) and (4.1.14) we find that the high energy vertex is given by

$$
\begin{aligned}
V & =\prod_{k=1}^{N}{ }_{(k)}\langle 0| \exp \left(-\sum_{i \neq j} \sum_{n=1}^{\infty}\left(\alpha_{2 n+2}^{\mu(i)} \frac{\mathcal{P}^{(2 n)}\left(z_{j}-z_{i}\right)}{(2 n+2) !} \alpha_{0 \mu}^{(j)}+\bar{\alpha}_{2 n+2}^{\mu(i)} \frac{\overline{\mathcal{P}}^{(2 n)}\left(\bar{z}_{j}-\bar{z}_{i}\right)}{(2 n+2) !} \alpha_{0 \mu}^{(j)}\right)\right. \\
& -\sum_{i} \sum_{n=1}^{\infty}\left(\left.\alpha_{2 n+2}^{\mu(i)} \frac{d^{2 n}}{d t^{2 n}} \frac{\left(\mathcal{P}(t)-t^{-2}\right)}{(2 n+2) !}\right|_{t=0} \alpha_{0 \mu}^{(i)}+\left.\bar{\alpha}_{2 n+2}^{\mu(i)} \frac{d^{2 n}}{d t^{2 n}} \frac{\left(\overline{\mathcal{P}}(t)-t^{-2}\right)}{(2 n+2) !}\right|_{t=0} \bar{\alpha}_{0 \mu}^{(i)}\right)
\end{aligned}
$$




$$
\left.+\sum_{i \neq j} \alpha_{0}^{\mu(i)} \ln \left|\sigma\left(z_{j}-z_{i}\right)\right| \alpha_{0 \mu}^{(j)}\right)
$$

The last term does not follow from the integrated overlap equations, but is derived from the unintegrated overlap involving $x^{\mu}$.

The high energy limit is given by extremising the vertex with respect to the KobaNielsen points and the moduli. The vertex will be an extremum with respect to the Koba-Nielsen coordinates if $\frac{\partial V}{\partial z_{i}}=0$. For the simple local coordinates which we are using equation (2.49), which is also valid at any genus and energy, implies that

$$
V L_{-1}^{(i)}=0
$$

In the high energy limit, $L_{-1}^{(i)}=\alpha_{-1}^{(i)} \cdot \alpha_{0}^{(i)}$ and using equation (4.1.14) this is automatically satisfied if the Koba-Nielsen points are at the half periods.

We now find the consequences of the vertex being at an extremum of the modulus of the surface. To this end we need to find which conformal transformation changes the moduli i.e. $w^{\prime} \rightarrow w^{\prime}+\epsilon$ as we have taken $2 w=1$. As such we need an $f$ in equation (2.25) such that

$$
f(z)=f(z+2 w), \quad f(z)=2+f\left(z+2 w^{\prime}\right)
$$

Such a function is given by

$$
f(z)=\frac{2 w}{i \pi}\left(\zeta(z)-\frac{\eta}{w} z\right)
$$

Substituting this function with argument $z-z_{i}$ into equation (2.27) we find in the high energy limit the equation

$$
\frac{i \pi}{2 w} \frac{\partial V}{\partial w^{\prime}}=V L_{-2}^{(i)}
$$

In deriving this equation we have used equation (4.1.16) and the fact that in the high energy limit $L_{n}^{(j)}=0, n \geq 0$ and $c_{f}$ vanishes as there is no $\alpha_{1} \cdot \alpha_{1}$ term in the vertex. The high energy vertex is an extremum and so

$$
V L_{-2}^{(i)}=0
$$

This equation and equation (4.1.16) ensure that any zero norm physical states decouple. Using equation (4.1.14), equation (4.1.20) implies in the high energy limit that

$$
V \alpha_{-2}^{(i)} \cdot \alpha_{0}^{(i)}=0
$$

Using equation (4.1.4), we can trade in $\alpha_{-2}^{(i)}$ to find that

$$
V \sum_{j, j \neq i} \mathcal{P}\left(z_{j}-z_{i}\right) \alpha_{0}^{(j)} \cdot \alpha_{0}^{(i)}=0
$$


Introducing $\mathcal{P}\left(z_{k}\right)=\varsigma_{k}$ the above equation becomes

$$
p_{1} \cdot p_{2} \varsigma_{3}+p_{1} \cdot p_{3} \varsigma_{2}+p_{1} \cdot p_{4} \varsigma_{1}=0
$$

and so

$$
\frac{s}{t}=\frac{p_{1} \cdot p_{2}}{p_{1} \cdot p_{3}}=-\frac{\left(\varsigma_{1}-\varsigma_{2}\right)}{\left(\varsigma_{1}-\varsigma_{3}\right)}
$$

where $s=-\left(p_{1}+p_{2}\right)^{2}, \quad t=-\left(p_{1}+p_{3}\right)^{2}, \quad u=-\left(p_{1}+p_{4}\right)^{2}$. As we explain in appendix $\mathrm{A}, \varsigma_{k}$ are the images of $z_{k}$ when the torus is mapped to the representation of the genus one surface that consists of the Riemann sphere with two branch cuts, denoted $\hat{C}_{2}$, and the points $\varsigma_{k}, k=1,2,3,4$ are the positions of the branch cuts. We note that $\varsigma_{4}=\infty$ and so the quantity that appears on the right-hand side is a cross ratio. Thus we recover the result of references $[2,3]$ found in the context of tachyon scattering.

We can also express the scattering of any physical states in terms of momenta multiplied by the tachyon amplitude. The discussion is much like that for the tree level given in section three. Any physical state is of the form

$$
\alpha_{-m_{1}}^{\mu_{1}} \ldots \alpha_{-m_{p}}^{\mu_{p}} \bar{\alpha}_{-n_{1}}^{\nu_{1}} \ldots \bar{\alpha}_{-n_{q}}^{\nu_{q}}|0, p\rangle \text {. }
$$

If the expression involves any odd moded oscillators then the amplitude will vanish using equations (4.1.6) and (4.1.14) in the extreme high energy limit. Using the overlaps relation of equation (4.1.4), the even moded oscillators can be traded for momenta. Once all the oscillators have been traded in this way one is left with an expression which is polynomial in momenta times the tachyon amplitude. However, as at tree level, the overlap relations are valid at all energies and resulting identities can be derived relating all scattering amplitudes to those of the tachyons.

Although the results found above for tachyon scattering agree with those found in references [2-5] those for other string states differ considerably. In particular in reference [5] the scattering of states containing odd moded oscillators does not vanish, but we see that there are no odd moded oscillators in the vertex and so these amplitudes vanish in the extreme high energy limit. The simplest example of this is the four graviton scattering which has a tree level contribution, but no one loop contribution.

For the open string we take $w^{\prime}=i \pi$ and $w=-\ln u$ where $u$ is a parameter which appears in the sewing of vertices to find the one loop amplitude. The planar one loop open string amplitude does not have an extremum at high energy, but the non-planar amplitude has with the Koba-Nielsen points at $0, w, w+w^{\prime}$ and $w^{\prime}$, that is at the half periods of the torus. The calculation for the open string proceeds along very similar lines to the closed string with almost all the equations being as above once one deletes any terms containing $\bar{\alpha}_{n}^{\mu}$ 's. For example, equations (4.1.1-4.1.15) hold. The vertex is given by equation (4.1.15) once one deletes the term containing $\bar{\alpha}_{n}^{\mu}$ 's. Equations (4.1.16-24) also hold with almost identical conclusions.

\subsection{Genus One Scattering using the Cut Sphere Representation}

We now repeat the calculation for the scattering of closed strings at genus one, but now representing the surface by the Riemann sphere with two square root branch cuts, 
denoted $\hat{C}_{2}$. The positions of the cuts are chosen to be between $\varsigma_{1}$ and $\varsigma_{2}$, and $\varsigma_{3}$ and $\varsigma_{4}=\infty$. The Fuchsian representation of the genus one surface, considered in the previous section, with coordinate $z$, and that of the Riemann sphere with two branch cuts and coordinate $\varsigma$ considered here are related by

$$
\mathcal{P}(z)=\varsigma, \quad z=\int_{\infty}^{\varsigma} \frac{d \varsigma^{\prime}}{Q\left(\varsigma^{\prime}\right)}
$$

where $Q=-2 \sqrt{\left(\varsigma-\varsigma_{1}\right)\left(\varsigma-\varsigma_{2}\right)\left(\varsigma-\varsigma_{3}\right)}=\mathcal{P}^{(1)}(z)$. A more detailed discussion of this mapping will be given in appendix A.

The high energy scattering is dominated by amplitudes for which the Koba-Nielsen points are at the half periods as in equation (4.1.5). Under the map to $\hat{C}_{2}$ these points get mapped to $\mathcal{P}\left(z_{i}\right)=\varsigma_{i}, i=1,2,3,4$, and are the beginning and end points of the branch cuts. They also sit on the real axis such that $\varsigma_{1}>\varsigma_{2}>\varsigma_{3}$.

The representation of the surface one uses does not by itself affect the form of the vertex in terms of oscillators as the overlap functions $\varphi$ used in one representation can be mapped using equation (4.2.1) to the other representation. However, the form of the vertex does depend on the local coordinates used. For string scattering using the Fuchsian representation we used the coordinate $z$ of the complex plane and the simple local coordinates $\xi_{i}=z-z_{i}$. Using equation (4.2.1), we find around the point $z_{i}$ that

$$
z-z_{i}=\int_{\varsigma_{i}}^{\varsigma} \frac{d \varsigma^{\prime}}{Q\left(\varsigma^{\prime}\right)}=\sum_{n=0}^{\infty} a_{2 n}^{(i)}\left(\varrho_{i}\right)^{2 n+1} .
$$

where

$$
\varrho_{i}=\sqrt{\left(\varsigma-\varsigma_{i}\right)}, i=1,2,3, \quad \varrho_{4}=\frac{1}{\sqrt{\varsigma}}
$$

For $i=1$, the lowest terms in this expansion are given by

$$
z-z_{1}=-\frac{\varrho_{1}}{\sqrt{\left(\varsigma_{1}-\varsigma_{2}\right)\left(\varsigma_{1}-\varsigma_{3}\right)}}+\frac{\varsigma_{1}}{2\left[\left(\varsigma_{1}-\varsigma_{2}\right)\left(\varsigma_{1}-\varsigma_{3}\right)\right]^{\frac{3}{2}}}\left(\varrho_{1}\right)^{3}+\ldots
$$

Equation (4.2.2) implies that $\varrho_{i}$ are good coordinates on $\hat{C}_{2}$ in the neighbourhood of the Koba-Nielsen points $\varsigma_{i}$. Indeed, they are related by an analytic transformation to the coordinates $\xi_{i}$. We note that $\varsigma-\varsigma_{i}$ are not good local coordinates as they fail to take account of the branch cuts. Indeed, they do not give a one to one map between the surface and a region of the complex plane as good coordinates are required to do. We have emphasised this point as it lies at the heart of the different results found in this paper compared to those of reference [5].

The two local coordinates $\xi_{i}$ and $\varrho_{i}$ are good local coordinates on the Fuchsian and $\hat{C}_{2}$ representations of the Riemann surface respectively. However, as they are related by an analytic transformation, we can use equation (4.2.2) to define a good set of $\xi_{i}$ local coordinates on $\hat{C}_{2}$ and, conversely, a good set of local coordinates $\varrho_{i}$ on the Fuchsian representation. Of course, the final scattering amplitude is independent of the local coordinates used, but the vertex and measure will be different. The overlap functions $\varphi$ used 
for the Fuchsian representation can be mapped over to $\hat{C}_{2}$ to yield overlap identities on $\hat{C}_{2}$. For example, if we work on $\hat{C}_{2}$, but use the coordinates $\xi_{i}$ to compute the vertex it will be exactly the same as the vertex computed using the Fuchsian representation with coordinates $\xi_{i}$ simply because the overlap functions have the same functional dependence on a given set of local coordinates regardless of which representation of the surface is used. When working on $\hat{C}_{2}$ it is more natural to use the local coordinates of equation (4.2.3) and in this section we will compute the vertex in this coordinate system. We note that because the coordinate transformation of equation (4.2.2) contains only odd powers of $\varrho_{i}$ the conformal map which relates the vertex $V$, computed with $\xi_{i}$ local coordinates, to the vertex, denoted $V_{\varrho}$, computed with $\varrho_{i}$ local coordinates, is given by

$$
V \prod_{i} \mathcal{N}_{i}^{(i)}=V_{\varrho}, \quad \text { where } \mathcal{N}_{i}^{(i)}=\exp \left(\sum_{n \geq 0} c_{2 n}^{(i)} L_{2 n}^{(i)}\right)
$$

Clearly, the functions

$$
\varphi_{c}=\frac{1}{\left(\varsigma-\varsigma_{i}\right)^{n}}=\frac{1}{\left(\varrho_{i}\right)^{2 n}}
$$

are well defined functions on $\hat{C}_{2}$, even if they are insensitive to the branch cuts. These lead to the overlap identities

$$
V_{\varrho}\left(\alpha_{-2 n}^{(i)}+\sum_{j, j \neq i} \frac{1}{\left(\varsigma_{j}-\varsigma_{i}\right)^{n}} \alpha_{0}^{(j)}\right)=0
$$

Other functions which are well defined on $\hat{C}_{2}$ are given by

$$
\varphi_{c}^{2 n-1} \equiv \frac{Q(\varsigma)}{\left(\varsigma-\varsigma_{i}\right)^{n}}=\frac{d_{(2 n-1)}}{\left(\varrho_{i}\right)^{2 n-1}}+\ldots+\frac{d_{1}}{\varrho_{i}}+\text { analytic, } \quad n \geq 2
$$

where $d_{i}$ are constants. The value $n=1$ is excluded as that function has a pole at infinity. In fact, equations (4.2.6) and (4.2.8) are all the possible functions defined on $\hat{C}_{2}$ with a single pole at one of the Koba-Nielsen points. They do indeed correspond to linear combinations of the overlap functions used in the previous section, as can be seen by using the relation [24]

$$
\mathcal{P}\left(z-z_{i}\right)=\varsigma_{i}+\frac{\left(\varsigma_{i}-\varsigma_{i+1}\right)\left(\varsigma_{i}-\varsigma_{i+2}\right)}{\varsigma-\varsigma_{i}}
$$

where $\varsigma_{i+3}=\varsigma_{i}$. Indeed, $\mathcal{P}^{(2 m-2)}\left(z-z_{1}\right)$ is a polynomial in $\mathcal{P}^{(2 m)}\left(z-z_{1}\right)$ and so in $\frac{1}{\left(\varsigma-\varsigma_{i}\right)^{m}}$, while

$$
\mathcal{P}^{(1)}\left(z-z_{1}\right)=-\frac{d \varsigma}{d z} \frac{\left(\varsigma_{i}-\varsigma_{i+1}\right)\left(\varsigma_{i}-\varsigma_{i+2}\right)}{\left(\varsigma-\varsigma_{i}\right)^{2}}=-\frac{\left(\varsigma_{i}-\varsigma_{i+1}\right)\left(\varsigma_{i}-\varsigma_{i+2}\right) Q(\varsigma)}{\left(\varsigma-\varsigma_{i}\right)^{2}} .
$$

Higher order odd derivatives are found using $\mathcal{P}^{(2 m+1)}\left(z-z_{1}\right)$ is a polynomial of degree $\mathrm{m}$ in $\mathcal{P}\left(z-z_{1}\right)$ times $\mathcal{P}^{(1)}$ and so all odd derivatives give superpositions of the functions in equation (4.2.8). 
To find an overlap equation involving $\alpha_{-1}^{\mu(i)}$, we require a function with a single first order pole. Such functions do not exist and so, as for the Fuchsian representation, we can instead use the function $\varphi_{1}$ of equation (4.1.11) expressed in terms of $\varrho_{i}$ coordinates. The integral of equation (4.1.10) over $B_{1}$ takes the same values since the vertices $V$ and $V_{\varrho}$ are related as in equation (4.2.5) and in the high energy limit we keep only terms involving momentum acting on the vertex. Evaluating the overlap we find that $V_{\varrho} \alpha_{-1}^{\mu(i)}=0$. This result follows more simply by carrying out the conformal transformation of equation (4.2.5) using equation (4.1.14). Using the overlap relations which arise from the functions of equation (4.2.8) we conclude that

$$
V_{\varrho} \alpha_{-(2 n+1)}^{\mu(i)}=0, \quad n \geq 0
$$

This equation and equation (4.2.7) imply that the vertex, using the coordinates $\varrho_{i}$, is given by

$$
\begin{gathered}
V_{\varrho}=\prod_{k=1}^{N}{ }_{(k)}\langle 0| \exp \left(-\sum_{i \neq j} \sum_{n=1}^{\infty}\left(\alpha_{2 n}^{\mu(i)} \frac{1}{2 n\left(\varsigma_{j}-\varsigma_{i}\right)^{n}} \alpha_{0 \mu}^{(j)}+\bar{\alpha}_{2 n}^{\mu(i)} \frac{1}{2 n\left(\bar{\varsigma}_{j}-\bar{\varsigma}_{i}\right)^{n}} \alpha_{0 \mu}^{(j)}\right)\right. \\
\left.+\sum_{i \neq j} \alpha_{0}^{\mu(i)} \ln \sqrt{\left|\left(\varsigma_{j}-\varsigma_{i}\right)\right|} \alpha_{0 \mu}^{(j)}\right)
\end{gathered}
$$

The last term in the exponential comes from the overlap identity $V_{\varrho} Q^{\mu(i)}\left(\varrho_{i}\right)=V_{\varrho} Q^{\mu(j)}\left(\varrho_{j}\right)$ and is in agreement with that found in references [2-5]. However, the rest of the vertex disagrees with that found in reference [5].

We observe that the vertex has a spectacularly simple form, and is just like that found at tree level in equation (3.2) except that all the odd moded oscillators are absent and we make the replacement $\left(z_{j}-z_{i}\right) \rightarrow \sqrt{\left(\varsigma_{j}-\varsigma_{i}\right)}$.

The extremum conditions on the vertex are much like that for the $\xi_{i}$ coordinates. Clearly, $V_{\varrho} L_{-1}^{(i)}=0$ and the reader will enjoy recovering equation (4.1.23) from the condition $V_{\varrho} L_{-2}^{(i)}=0$ using equation $(4.2 .7)$ for $n=1$. We note that this last calculation is much like the analogous calculation at tree level.

The results for the open string essentially follow the same path as explained in the previous section.

We now comment on the relation of the results contained in this paper with those found in references [2-5]. The four tachyon scattering at high energy found here agrees precisely with that found in references [2-4]. While almost all of references [2-4] concentrate on tachyon scattering, reference [5] gives genus independent relations between an arbitrary string scattering amplitude and that for the same number of tachyons. The results for arbitrary string scattering found here disagree with those found in reference [5]. Indeed, some of the amplitudes, such as that for graviton scattering, discussed in [5] are found to vanish at one loop, but are non-zero at tree level, and as a result we do not find the genus independent relations found in reference [5]. 
Reference [5] uses the sum over world histories approach within the context of the representation of the string world-surface by the Riemann sphere with two branch cuts of degree $g$, where $g$ is the genus of the surface. By degree $g$ we mean that the two branch cuts are of the form $(z)^{\frac{1}{g+1}}$, and so lead to a surface with $g+1$ different sheets. For genus one this is the representation of the torus with two square root branch cuts used above. While we agree with reference [5] that the part of the vertex which depends only on the momenta can be expressed in terms of the positions of the branch cuts written in terms of the coordinate $\varsigma$ of the Riemann sphere with two branch cuts, the source of our disagreement stems from the use of such a coordinate to describe the behaviour of the other physical states at high energy. It is important to remember that the high energy behaviour is dominated when the Koba-Nielsen points are at the beginning and end of the branch cuts. As spelt out below equation (4.2.4) $\varsigma-\varsigma_{i}$ are not a good set of local coordinates to use when considering arbitrary string scattering from points that are at the beginning and end of the branch cuts, as they do not take account of the branch cuts there. As a result, they can not be used to formulate the scattering of arbitrary string states at high energy as was done in reference [5].

\section{The Purely Momentum Dependence of String Vertices.}

In this section, we will compute the purely momentum dependence of the closed and open string vertices (i.e the part of the vertex involving only $\alpha_{0}^{\mu}$ ) in terms of the coefficients that occur in the rest of the vertex. We do this in such a way that it is valid for the vertex at any genus with any number of strings scattering. The purely momentum piece of the vertex for planar open string scattering at any genus was found in $[13,14]$.

The part of the vertex involving non-zero modes (i.e. $\alpha_{n}^{\mu}, n \geq 1$ ) can be deduced using the integrated overlap equations. This has been carried out [13,14] for all genus vertices for planar open string scattering and the computation for the closed string vertices is similar. In section six we will do the computation for the closed string and non-planar open string genus one vertices. We can write the vertex in the form

$$
\begin{aligned}
V^{\text {closed }} & =\prod_{k=1}^{N}{ }_{(k)}\langle 0| \exp \left(-\sum_{i, j}\left\{\frac{1}{2} \sum_{n, m=1}^{\infty} \alpha_{n}^{\mu(i)} N_{n m}^{i j} \alpha_{m \mu}^{(j)}+\sum_{n=1}^{\infty} \alpha_{n}^{\mu(i)} N_{n}^{i j} \alpha_{0 \mu}^{(j)}\right.\right. \\
& \left.\left.+\left(N_{n m}^{i j} \rightarrow \bar{N}_{n m}^{i j}, N_{n}^{i j} \rightarrow \bar{N}_{n}^{i j}, \alpha_{n}^{\mu(k)} \rightarrow \bar{\alpha}_{n}^{\mu(k)}\right)\right\}\right) V_{00}^{\text {closed }}
\end{aligned}
$$

Now we want to find the zero mode piece, which we write

$$
V_{00}^{\text {closed }}=\exp \left(-\frac{1}{2} \sum_{i, j} \alpha_{0}^{\mu(i)} N^{i j} \alpha_{0 \mu}^{(j)}\right) \text {. }
$$

The purely momentum piece can not be calculated from the integrated overlap equations as they involve no operator ( i.e $q^{\mu}$ ) that is sensitive to this term. To calculate the $N^{i j}$ we 
need to use the overlap with $x^{\mu}$. The mode expansion of the embedding field, denoted $x^{\mu}$, can be written as

$$
x^{\mu}(z, \bar{z})=q^{\mu}-i \frac{\alpha^{\prime}}{2} p^{\mu} \ln |z|^{2}+i \sqrt{\frac{\alpha^{\prime}}{2}} \sum_{n \neq 0} \frac{1}{n}\left(\alpha_{n}^{\mu} z^{-n}+\bar{\alpha}_{n}^{\mu} \bar{z}^{-n}\right) .
$$

We have $\alpha_{0}^{\mu}=\bar{\alpha}_{0}^{\mu}=\sqrt{\frac{\alpha^{\prime}}{2}} p^{\mu}$ and, from the canonical commutation relation $\left[p^{\mu}, q^{\nu}\right]=$ $-i \eta^{\mu \nu}$, we can write

$$
q^{\mu}=-i \sqrt{\frac{\alpha^{\prime}}{2}} \frac{\overleftarrow{\partial}}{\partial \alpha_{0 \mu}}
$$

and we can therefore factor out the universal $i \sqrt{\frac{\alpha^{\prime}}{2}}$ factor and write

$$
x^{\mu}(z, \bar{z})=i \sqrt{\frac{\alpha^{\prime}}{2}}\left(-\frac{\overleftarrow{\partial}}{\partial \alpha_{0 \mu}}-\alpha_{0}^{\mu} \ln |z|^{2}+\sum_{n \neq 0} \frac{1}{n} \alpha_{n}^{\mu} z^{-n}+\sum_{n \neq 0} \frac{1}{n} \bar{\alpha}_{n}^{\mu} \bar{z}^{-n}\right) .
$$

The unintegrated overlap for $x^{\mu}$ is given by

$$
V x^{\mu(i)}\left(\xi_{i}, \bar{\xi}_{i}\right)=V x^{\mu(j)}\left(\xi_{j}, \bar{\xi}_{j}\right)
$$

where $V$ is given by (5.1) and (5.2), and we choose the local coordinates $\xi_{i}=z-z_{i}$ where $z$ is the coordinate on the Fuchsian or Schottky representation of the Riemann surface. For simplicity, we will not write anymore the spacetime indices as it is obvious how they appear. The overlap equation (5.6), using equation (5.5) and the form of the vertex of equation (5.1), becomes

$$
\begin{gathered}
V\left\{\sum_{k} N^{i k} \alpha_{0}^{(k)}+\sum_{n=1}^{\infty} \sum_{k}\left(\alpha_{n}^{(k)} N_{n}^{k i}+\bar{\alpha}_{n}^{(k)} \bar{N}_{n}^{k i}\right)-\alpha_{0}^{(i)} \ln \left|\xi_{i}\right|^{2}\right. \\
+\sum_{n, m=1}^{\infty} \sum_{k}\left(N_{n m}^{i k}\left(\xi_{i}\right)^{n} \alpha_{m}^{(k)}+\bar{N}_{n m}^{i k}\left(\bar{\xi}_{i}\right)^{n} \bar{\alpha}_{m}^{(k)}\right)+\sum_{n=1}^{\infty} \sum_{k}\left(N_{n}^{i k} \alpha_{0}^{(k)}\left(\xi_{i}\right)^{n}+\bar{N}_{n}^{i k} \bar{\alpha}_{0}^{(k)}\left(\bar{\xi}_{i}\right)^{n}\right) \\
\left.+\sum_{n=1}^{\infty}\left(\frac{1}{n} \alpha_{n}^{(i)}\left(\xi_{i}\right)^{-n}+\frac{1}{n} \bar{\alpha}_{n}^{(i)}\left(\bar{\xi}_{i}\right)^{-n}\right)\right\}=V\{i \leftrightarrow j\},
\end{gathered}
$$

where the right-hand side is obtained from the left-hand side by changing all $i$ 's by $j$ 's.

Let us start by considering only the $\alpha_{0}$ terms, and assume that $N_{n}^{i k}$ has the form

$$
\begin{gathered}
N_{n}^{i k}=-\left.\frac{(-1)^{n}}{n !} \frac{d^{n}}{d t^{n}}\left(\ln \psi\left(z_{k}-z_{i}+t\right)\right)\right|_{t=0} \quad k \neq i \\
N_{n}^{i i}=-\left.\frac{(-1)^{n}}{n !} \frac{d^{n}}{d t^{n}}(\ln \psi(t)-\ln t)\right|_{t=0},
\end{gathered}
$$


and similarly for $\bar{N}_{n}^{i k}$, but with $\psi(z)$ replaced by $\bar{\psi}(\bar{z})$, and that $(\ln \psi(z)-\ln z)$ is analytic in a neighborhood of $z=0$. These relations are a consequence of the integrated overlap identities that determine the non-zero mode part of the vertex. Indeed, $-\left.\frac{(-1)^{n}}{n !} \frac{d^{n}}{d t^{n}}\left(\ln \psi\left(z-z_{i}+t\right)-\ln \left(z-z_{i}+t\right)\right)\right|_{t=0}$ for $n \geq 1$ are closely related to the functions used in the overlap relations for the operators $\partial x^{\mu}$. The assumptions (5.8) allow us to write the second line of (5.7) in a simple manner

$$
\begin{aligned}
\sum_{n=1}^{\infty} N_{n}^{i k}\left(\xi_{i}\right)^{n}=- & \left.\sum_{n=1}^{\infty} \frac{\left(-\xi_{i}\right)^{n}}{n !} \frac{d^{n}}{d t^{n}}\left(\ln \psi\left(z_{k}-z_{i}+t\right)\right)\right|_{t=0}=\ln \left(\frac{\psi\left(z_{k}-z_{i}\right)}{\psi\left(z_{k}-z\right)}\right) \quad k \neq i \\
& \sum_{n=1}^{\infty} \xi_{i}^{n} N_{n}^{i i}=-\ln \left(\frac{\psi\left(-\xi_{i}\right)}{-\xi_{i}}\right)+\lim _{t \rightarrow 0} \ln \left(\frac{\psi(t)}{t}\right)
\end{aligned}
$$

and similarly for the complex conjugates. The overlap (5.7) thus becomes, for the $\alpha_{0}$ terms

$$
\begin{gathered}
V\left\{\sum_{k} N^{i k} \alpha_{0}^{(k)}+\sum_{k, k \neq i} \ln \left|\frac{\psi\left(z_{k}-z_{i}\right)}{\psi\left(z_{k}-z\right)}\right|^{2} \alpha_{0}^{(k)}\right. \\
\left.+\left(-\ln \left|\xi_{i}\right|^{2}-\ln \left|\frac{\psi\left(-\xi_{i}\right)}{-\xi_{i}}\right|^{2}+\lim _{t \rightarrow 0} \ln \left|\frac{\psi(t)}{t}\right|^{2}\right) \alpha_{0}^{(i)}\right\}=V\{i \leftrightarrow j\} .
\end{gathered}
$$

We see that the $-\ln \left|\psi\left(z_{k}-z\right)\right|^{2}$ terms cancel with the right-hand side, except for $k=j$, which we can trade with the right-hand side for $\ln \left|\psi\left(z_{i}-z\right)\right|^{2}=\ln \left|\psi\left(-\xi_{i}\right)\right|^{2}$ which simplifies with the same term in the second line. We are thus left with

$$
V\left\{\sum_{k} N^{i k} \alpha_{0}^{(k)}+\sum_{k, k \neq i} \ln \left|\psi\left(z_{k}-z_{i}\right)\right|^{2} \alpha_{0}^{(k)}+\lim _{t \rightarrow 0} \ln \left|\frac{\psi(t)}{t}\right|^{2} \alpha_{0}^{(i)}\right\}=V\{i \leftrightarrow j\}
$$

Thus we find our desired result that the purely momentum part of the vertex is given by

$$
N^{i k}= \begin{cases}-\ln \left|\psi\left(z_{k}-z_{i}\right)\right|^{2} & i \neq k \\ -\lim _{t \rightarrow 0} \ln \left|\frac{\psi(t)}{t}\right|^{2} & i=k\end{cases}
$$

Now let us consider the $\alpha_{m}^{k}$ terms in (5.7). We make the further assumption

$$
\begin{gathered}
N_{n m}^{i k}=-\left.\frac{(-1)^{n}}{n !} \frac{d^{n}}{d t^{n}} F_{m}\left(z_{k}-z_{i}+t\right)\right|_{t=0} \quad k \neq i \\
N_{n m}^{i i}=-\left.\frac{(-1)^{n}}{n !} \frac{d^{n}}{d t^{n}}\left(F_{m}(t)+\frac{1}{m} \frac{1}{(-t)^{m}}\right)\right|_{t=0},
\end{gathered}
$$


with analogous equations for $\bar{N}_{n m}^{i k}$. These relations also follow from the integrated overlap relations. Therefore we can write

$$
\begin{gathered}
V\left\{-\sum_{m=1}^{\infty} \sum_{k, k \neq i} \alpha_{m}^{(k)}\left(\left.\frac{(-1)^{m}}{m !} \frac{d^{m}}{d t^{m}}\left(\ln \psi\left(z_{i}-z_{k}+t\right)\right)\right|_{t=0}+F_{m}\left(z_{k}-z\right)-F_{m}\left(z_{k}-z_{i}\right)\right)\right. \\
\left.-\sum_{m=1}^{\infty} \alpha_{m}^{(i)}\left(F_{m}\left(-\xi_{i}\right)-\lim _{t \rightarrow 0}\left(F_{m}(t)+\frac{1}{m} \frac{1}{(-t)^{m}}\right)+\left.\frac{(-1)^{m}}{m !} \frac{d^{m}}{d t^{m}} \ln \left(\frac{\psi(t)}{t}\right)\right|_{t=0}\right)\right\} \\
=V\{i \leftrightarrow j\}
\end{gathered}
$$

where, in the first line, we have used that

$$
\left.\sum_{n=1}^{\infty} \frac{(-1)^{n}}{n !}\left(\xi_{i}\right)^{n} \frac{d^{n}}{d t^{n}} F_{m}\left(z_{k}-z_{i}+t\right)\right|_{t=0}=F_{m}\left(z_{k}-z\right)-F_{m}\left(z_{k}-z_{i}\right)
$$

And similarly for the second line. We see that the $F_{m}\left(z_{k}-z\right)$ terms with $k \neq i, j$, simplify with the right-hand side, and the remaining terms will cancel with the $F_{m}\left(-\xi_{i}\right)$ in the second line. We are thus left with

$$
\begin{gathered}
V\left\{-\sum_{m=1}^{\infty} \sum_{k, k \neq i} \alpha_{m}^{(k)}\left(\left.\frac{(-1)^{m}}{m !} \frac{d^{m}}{d t^{m}}\left(\ln \psi\left(z_{i}-z_{k}+t\right)\right)\right|_{t=0}-F_{m}\left(z_{k}-z_{i}\right)\right)\right. \\
\left.-\sum_{m=1}^{\infty} \alpha_{m}^{(i)}\left(-\lim _{t \rightarrow 0}\left(F_{m}(t)+\frac{1}{m} \frac{1}{(-t)^{m}}\right)+\left.\frac{(-1)^{m}}{m !} \frac{d^{m}}{d t^{m}} \ln \left(\frac{\psi(t)}{t}\right)\right|_{t=0}\right)\right\} \\
=V\{i \leftrightarrow j\} .
\end{gathered}
$$

This equation is satisfied if

$$
F_{m}(z)=\left.\frac{1}{m !} \frac{d^{m}}{d t^{m}} \ln \psi(-(z+t))\right|_{t=0}
$$

and therefore

$$
N_{n m}^{i k}= \begin{cases}-\left.\frac{(-1)^{m}}{n ! m !} \frac{d^{n+m}}{d t^{n+m}} \ln \psi\left(z_{i}-z_{k}+t\right)\right|_{t=0} & i \neq k \\ -\left.\frac{(-1)^{m}}{n ! m !} \frac{d^{n+m}}{d t^{n+m}}(\ln \psi(t)-\ln t)\right|_{t=0} & i=k\end{cases}
$$

For the open string we use the operator

$$
Q^{\mu}(z)=q^{\mu}-i \sqrt{2 \alpha^{\prime}} \alpha_{0}^{\mu} \ln |z|+i \sqrt{2 \alpha^{\prime}} \sum_{n \neq 0} \frac{1}{n} \alpha_{n}^{\mu} z^{-n} .
$$


We now have $\alpha_{0}^{\mu}=\sqrt{2 \alpha^{\prime}} p^{\mu}$, and using the canonical commutation relation $\left[p^{\mu}, q^{\nu}\right]=-i \eta^{\mu \nu}$, we can write

$$
q^{\mu}=-i \sqrt{2 \alpha^{\prime}} \frac{\overleftarrow{\partial}}{\partial \alpha_{0 \mu}}
$$

and we can therefore factor out the universal $i \sqrt{2 \alpha^{\prime}}$ factor and write

$$
Q^{\mu}(z)=i \sqrt{2 \alpha^{\prime}}\left(-\frac{\overleftarrow{\partial}}{\partial \alpha_{0 \mu}}-\alpha_{0}^{\mu} \ln |z|^{2}+\sum_{n \neq 0} \frac{1}{n} \alpha_{n}^{\mu} z^{-n}\right)
$$

The operator $Q^{\mu}(z)$ has conformal weight one, but it is not the same as the field $x^{\mu}$ for the open string which depends on $z$ and $\bar{z}$. Rather, it is the field $x^{\mu}(\tau, 0)$ with $z=e^{\tau}$, extended by analytic continuation into the upper half plane. And if the surface is doubled, also into the lower half plane by reflection on the real axis. Of course, before taking these steps we have mapped the field from the string world-sheet to the upper half plane and rotated to Euclidean space.

The calculation of the purely momentum piece of the vertex proceeds much as for the closed string if one just drops most of the anti-holomorphic terms and terms containing $\bar{\alpha}_{n}$. We can write the vertex as

$$
\begin{aligned}
V^{\text {open }}=\prod_{k=1}^{N}{ }_{(k)}\langle 0| \exp ( & \left.-\sum_{i, j}\left\{\frac{1}{2} \sum_{n, m=1}^{\infty} \alpha_{n}^{\mu(i)} N_{n m}^{i j} \alpha_{m \mu}^{(j)}+\sum_{n=1}^{\infty} \alpha_{n}^{\mu(i)} N_{n}^{i j} \alpha_{0 \mu}^{(j)}\right\}\right) \\
& \exp \left(-\frac{1}{2} \sum_{i, j} \alpha_{0}^{\mu(i)} N^{i j} \alpha_{0 \mu}^{(j)}\right) .
\end{aligned}
$$

We are using the same symbols for the coefficients in the open string vertex, the reader will know from the context which string is being considered. We begin from the overlap relations

$$
V Q^{\mu(i)}\left(\xi_{i}\right)=V Q^{\mu(j)}\left(\xi_{j}\right)
$$

and assume that $N_{n}^{i k}$ has the form

$$
\begin{gathered}
N_{n}^{i k}=-\left.\frac{(-1)^{n}}{n !} \frac{d^{n}}{d t^{n}}\left(\ln \psi\left(z_{k}-z_{i}+t\right)\right)\right|_{t=0} \quad k \neq i \\
N_{n}^{i i}=-\left.\frac{(-1)^{n}}{n !} \frac{d^{n}}{d t^{n}}(\ln \psi(t)-\ln t)\right|_{t=0},
\end{gathered}
$$

Comparing the $\alpha_{0}$ terms in equation (5.22) we find that the purely momentum part of the vertex is given by

$$
N^{i k}= \begin{cases}-\ln \left|\psi\left(z_{k}-z_{i}\right)\right| & i \neq k \\ -\lim _{t \rightarrow 0} \ln \left|\frac{\psi(t)}{t}\right| & i=k\end{cases}
$$


While comparing the $\alpha_{n}, n>0$ and assuming that

$$
\begin{gathered}
N_{n m}^{i k}=-\left.\frac{(-1)^{n}}{n !} \frac{d^{n}}{d t^{n}} F_{m}\left(z_{k}-z_{i}+t\right)\right|_{t=0} \quad k \neq i \\
N_{n m}^{i i}=-\left.\frac{(-1)^{n}}{n !} \frac{d^{n}}{d t^{n}}\left(F_{m}(t)+\frac{1}{m} \frac{1}{(-t)^{m}}\right)\right|_{t=0},
\end{gathered}
$$

we find that equation (5.22) is satisfied if

$$
F_{m}(z)=\left.\frac{1}{m !} \frac{d^{m}}{d t^{m}} \ln \psi(-(z+t))\right|_{t=0}
$$

In fact equations (5.23) and (5.25) follow from the integrated overlap equations. As a result, we find that

$$
N_{n m}^{i k}= \begin{cases}-\left.\frac{(-1)^{m}}{n ! m !} \frac{d^{n+m}}{d t^{n+m}} \ln \psi\left(z_{i}-z_{k}+t\right)\right|_{t=0} & i \neq k \\ -\left.\frac{(-1)^{m}}{n ! m !} \frac{d^{n+m}}{d t^{n+m}}(\ln \psi(t)-\ln t)\right|_{t=0} & i=k\end{cases}
$$

\section{One Loop Open and Closed Arbitrary String Scattering}

In references $[13,14]$ the planar open string multi-string scattering vertex and measure

were computed. In the first subsection we first calculate the one loop multi-string vertex for closed string scattering and in the next subsection the measure. Finally we do the same for non-planar open string scattering.

\subsection{The nonzero mode part of the Closed Vertex}

We use the Fuchsian representation of the Riemann surface as explained at the beginning of section four, and we will adopt the local coordinates $\xi_{i}=z-z_{i}$. For the closed string we take $w^{\prime}$ arbitrary but $2 w=1$, however, as similar calculations are useful for the open string we will sometimes keep factors of $w$ explicitly rather than set it equal to $\frac{1}{2}$. As explained in section two we may use the integrated overlap identities of equation (2.15) for the conformal operator $\partial x^{\mu}$ to determine much of the string vertex. These identities require scalar functions $\varphi$ which are defined on the torus and so in our representation of the torus obey

$$
\varphi(z)=\varphi(z+2 w), \quad \varphi(z)=\varphi\left(z+2 w^{\prime}\right)
$$

and analytic except for possible poles at the Koba-Nielsen points. The identities are most useful for functions that have poles at only one Koba-Nielsen point. A complete set of such functions are given by the Weierstrass function $\mathcal{P}$ and its derivatives, and so we take

$$
\varphi_{n}\left(\xi_{i}\right)=\frac{(-1)^{n}}{(n) !} \mathcal{P}^{(n-2)}\left(\xi_{i}\right)=\left\{\begin{array}{l}
\frac{1}{n\left(\xi_{i}\right)^{n}}+N_{n}^{i i}+\sum_{m=1} N_{n m}^{i i}\left(\xi_{i}\right)^{m} \\
N_{n}^{i j}+\sum_{m=1} N_{n m}^{i j}\left(\xi_{j}\right)^{m}
\end{array} \quad n \geq 2 .\right.
$$


The overlap equation is then given by

$$
V\left(\frac{1}{n} \alpha_{-n}^{\mu(i)}+\sum_{j} N_{n}^{i j} \alpha_{0}^{\mu(j)}+\sum_{j} \sum_{m=1}^{\infty} N_{n m}^{i j} \alpha_{m}^{\mu(j)}\right)=0, \quad n \geq 2 .
$$

We note that, for $n \geq 2$

$$
\begin{gathered}
N_{n m}^{i j}=\left.\frac{(-1)^{n}}{n ! m !} \frac{d^{(n+m-2)} \mathcal{P}\left(z_{j}-z_{i}+t\right)}{d t^{(n+m-2)}}\right|_{t=0}=\left.\frac{(-1)^{m}}{n ! m !} \frac{d^{(n+m-2)} \mathcal{P}\left(z_{i}-z_{j}+t\right)}{d t^{(n+m-2)}}\right|_{t=0}, i \neq j \\
N_{n m}^{i i}=\left.\frac{(-1)^{n}}{n ! m !} \frac{d^{(n+m-2)}\left(\mathcal{P}(t)-\frac{1}{t^{2}}\right)}{d t^{(n+m-2)}}\right|_{t=0} \\
N_{n}^{i j}=\left.\frac{(-1)^{n}}{n !} \frac{d^{(n-2)} \mathcal{P}\left(z_{j}-z_{i}+t\right)}{d t^{(n-2)}}\right|_{t=0}, i \neq j \\
N_{n}^{i i}=\left.\frac{(-1)^{n}}{n !} \frac{d^{(n-2)}\left(\mathcal{P}(t)-t^{-2}\right)}{d t^{(n-2)}}\right|_{t=0} .
\end{gathered}
$$

There is no scalar function with a pole of order one at a single point, and so at first sight we do not have an integrated overlap identity that can determine the dependence of the vertex on $\alpha_{1}^{\mu}$. The solution to this difficulty is to consider functions which are not periodic, but shift by constants under the change of periods, namely a function such that

$$
\varphi(z+2 w)=\varphi(z)+c_{1}, \quad \varphi\left(z+2 w^{\prime}\right)=\varphi(z)+c_{2}
$$

We now consider the contour integral of $V \partial x^{\mu} \varphi$ around the boundary of the parallelogram that describes the torus. The contributions from opposing sides no longer cancel as $\varphi$ is no longer defined on the torus and one finds, instead of equation (2.15), that

$$
\sum_{j} \oint d \xi_{j} V \varphi \partial x^{\mu(j)}\left(\xi_{j}\right)=c_{2} \int_{B_{1}} V \partial x^{\mu} d z+c_{1} \int_{A_{2}} V \partial x^{\mu} d z
$$

where the paths $B_{1}$ and $A_{2}$ are shown in figure 1 . On the right hand side $V \partial x^{\mu} d z$ stands for $V \partial x^{\mu(k)}\left(\xi_{k}\right) d \xi_{k}$, which is independent of which string Hilbert space one uses by virtue of equation (2.5) using the operator $\partial x^{\mu}$. It should not matter which function $\varphi$ one takes as long as it obeys equation (6.1.5). Functions of this type, with a pole of order one at the same place, differ by a constant times $z$. Taking $\varphi=z$ in equation (6.1.6) we find that provided

$$
w^{\prime} \int_{B_{1}} V \partial x^{\mu} d z+w \int_{A_{2}} V \partial x^{\mu} d z=\frac{1}{2} V\left(\sum_{j} z_{j} \alpha_{0}^{\mu(j)}+\sum_{j} \alpha_{1}^{\mu(j)}\right)
$$

we will indeed find the same result no matter what function $\varphi$ we take. 
Equation (6.1.7) does not determine the integrals along the $B_{1}$ and $A_{2}$ segments of the parallelogram, but as we will argue later, the unique solution taking into account modular invariance, is given by

$$
\begin{gathered}
\int_{B_{1}} V \partial x^{\mu} d z=\frac{\bar{w}}{\left(w^{\prime}-\bar{w}^{\prime}\right)} V \sum_{j} \alpha_{1}^{(j)}-V \sum_{j} \frac{\left(w \bar{z}_{j}-\bar{w} z_{j}\right)}{\left(w^{\prime}-\bar{w}^{\prime}\right)} \alpha_{0}^{\mu(j)} \\
\int_{A_{2}} V \partial x^{\mu} d z=-\frac{\bar{w}^{\prime}}{\left(w^{\prime}-\bar{w}^{\prime}\right)} V \sum_{j} \alpha_{1}^{(j)}+V \sum_{j} \frac{\left(w^{\prime} \bar{z}_{j}-\bar{w}^{\prime} z_{j}\right)}{\left(w^{\prime}-\bar{w}^{\prime}\right)} \alpha_{0}^{\mu(j)} .
\end{gathered}
$$

We observe that under the exchange $w \leftrightarrow w^{\prime}$ the two integrals are exchanged with minus sign provided we recognise that $w^{\prime}-\bar{w}^{\prime}=2 i \operatorname{Im} w^{\prime}=i \operatorname{Im} \tau$ is $i$ times the area of the fundamental region and is unchanged in this process.

The function

$$
\varphi_{1}(z)=\zeta(z)-\frac{\eta}{w} z
$$

obeys equation (6.1.5) with $c_{1}=0$ and $c_{2}=-\frac{i \pi}{w}$. In this equation $\zeta(z)$ is the zeta function which is defined to obey $-\frac{d \zeta(z)}{d z}=\mathcal{P}(z)$ and has a first order pole with residue one. It is not a periodic function, but obeys

$$
\zeta(z+2 w)=\zeta(z)+2 \eta, \quad \zeta\left(z+2 w^{\prime}\right)=\zeta(z)+2 \eta^{\prime}
$$

where $\eta$ and $\eta^{\prime}$ are independent of $z$. See appendix A for more details. Substituting $\varphi_{1}$ into equation (6.1.6) and using equation (6.1.8) we find that

$$
\begin{gathered}
V\left(\alpha_{-1}^{\mu(i)}+\sum_{j} \sum_{m=1}^{\infty} \tilde{N}_{1 m}^{i j} \alpha_{m}^{\mu(j)}+\sum_{j} \tilde{N}_{1}^{i j} \alpha_{0}^{\mu(j)}\right) \\
=-\frac{i \pi}{w} V\left(\frac{\bar{w}}{\left(w^{\prime}-\bar{w}^{\prime}\right)} \sum_{j} \alpha_{1}^{(j)}-\sum_{j} \frac{\left(w \bar{z}_{j}-\bar{w} z_{j}\right)}{\left(w^{\prime}-\bar{w}^{\prime}\right)} \alpha_{0}^{\mu(j)}\right) .
\end{gathered}
$$

The coefficients in this equation are just those found by expanding $\varphi_{1}$ around the Koba-Nielsen points in terms of the local coordinates $\xi_{i}$, indeed

$$
\varphi_{1}\left(z-z_{i}\right)=\left\{\begin{array}{l}
\frac{1}{\xi_{i}}+\sum_{m=1} \tilde{N}_{1 m}^{i i}\left(\xi_{i}\right)^{m} \\
\tilde{N}_{1}^{i j}+\sum_{m=1} \tilde{N}_{1 m}^{i j}\left(\xi_{j}\right)^{m} .
\end{array}\right.
$$

Clearly, the coefficients are given by

$$
\begin{gathered}
\tilde{N}_{1 m}^{i j}=\left.\frac{1}{m !} \frac{d^{m} \varphi_{1}\left(z_{j}-z_{i}+t\right)}{d t^{m}}\right|_{t=0}, \quad \tilde{N}_{1}^{i j}=\varphi_{1}\left(z_{j}-z_{i}\right), \quad i \neq j \\
\tilde{N}_{1 m}^{i i}=\left.\frac{1}{m !} \frac{d^{m}}{d t^{m}}\left(\varphi_{1}(t)-\frac{1}{t}\right)\right|_{t=0}
\end{gathered}
$$


From the integrated overlap equations (6.1.3) and (6.1.11) we read off the vertex to be

$$
\begin{gathered}
V^{\text {closed }}=\prod_{k=1}^{N}{ }_{(k)}\langle 0| \exp \left(-\sum_{i, j}\left\{\frac{1}{2} \sum_{n, m=1}^{\infty} \alpha_{n}^{\mu(i)} N_{n m}^{i j} \alpha_{m \mu}^{(j)}+\sum_{n=1}^{\infty} \alpha_{n}^{\mu(i)} N_{n}^{i j} \alpha_{0 \mu}^{(j)}\right.\right. \\
\left.\left.+\left(N_{n m}^{i j} \rightarrow \bar{N}_{n m}^{i j}, N_{n}^{i j} \rightarrow \bar{N}_{n}^{i j}, \alpha_{n}^{\mu(k)} \rightarrow \bar{\alpha}_{n}^{\mu(k)}\right)\right\}\right) \exp \left(-\frac{1}{2} \sum_{i, j} \alpha_{0}^{\mu(i)} N^{i j} \alpha_{0 \mu}^{(j)}\right) .
\end{gathered}
$$

The coefficients $N_{n m}^{i j}$ and $N_{n}^{i j}$ for $n \geq 2$ are given in equation (6.1.4), while the remainders are

$$
N_{1 m}^{i j}=\tilde{N}_{1 m}^{i j}+\frac{\pi i}{\left(w^{\prime}-\bar{w}^{\prime}\right)} \delta_{1, m}, \quad N_{1}^{i j}=\tilde{N}_{1}^{i j}-\frac{2 \pi \operatorname{Im}\left(z_{j}-z_{i}\right)}{\left(w^{\prime}-\bar{w}^{\prime}\right)}
$$

The additions to the latter coefficients, and so to the vertex, arise from the contributions on the right hand side of equation (6.1.11) which in turn are a consequence of the non-periodic nature of $\varphi_{1}$.

The zero mode piece can be read off from section five equation (5.12) in terms of the coefficients that appear in the vertex. In deriving this result, we made some assumptions about the coefficients and we now show that these are a consequence of the results above. Let us consider the function

$$
\ln \psi(z)=\left(\ln \sigma(z)-\frac{\eta}{2 w} z^{2}\right)+c . c-\frac{2 \pi}{\operatorname{Im} \tau}(\operatorname{Im} z)^{2}=\ln \frac{\theta_{1}(v)}{\theta_{1}^{\prime}(0)}+c . c-\frac{2 \pi}{\operatorname{Im} \tau}(\operatorname{Im} z)^{2} .
$$

The $\sigma$ function is defined by

$$
\zeta(z)=\frac{d}{d z} \ln \sigma(z) \quad, \quad \mathcal{P}(z)=-\frac{d \zeta(z)}{d z}
$$

and in the last step of equation (6.1.16) we have used the relation between the $\sigma$ function and the $\theta_{1}$ function to give an alternative expression. The argument of the theta functions is $v=\frac{z}{2 w}$, but it also depends on $\tau$. We observe that the function is not a holomorphic function of $z$ only as a consequence of the last term. As such, when taking the derivatives we use the expression

$$
\frac{d}{d z}=\frac{1}{2}\left(\frac{d}{d z_{1}}-i \frac{d}{d z_{2}}\right)
$$

where $z=z_{1}+i z_{2}$. Calculating the coefficients using equations (5.8) and equations (5.17) we do find precisely the results of equations (6.1.4), (6.1.13) and (6.1.15). This is rather obvious for terms which have more than two derivatives as the last two terms in equation (6.1.16) do not contribute. This only leaves one and two derivatives which is easily carried out. One finds that the non-holomorphic dependence of $\ln \psi$ arises from the the contour integral along $B_{1}$ of equation (6.1.11). Thus we find, as for all string vertices, that it is determined by only one function $\ln \psi$. One can think of this as a consequence of the definition of the vertex in terms of overlap relations. 


\subsection{The measure for the Closed Vertex}

As explained in section two the measure is determined by the decoupling of zero norm null physical states. In the simple coordinate system in which we are working, i.e. $\xi_{i}=z-z_{i}$, at genus one, and indeed for all genus, one finds that

$$
V L_{-1}^{(i)}=\frac{\partial V}{\partial z_{i}}
$$

The explanation is the same as for the case of tree level in equation (2.49). Following the discussion below this equation, that is equations (2.50) and (2.51) we conclude that the measure $f_{m}$ is independent of $z_{i}$.

Decoupling of the zero norm physical states at higher levels requires us to understand how a conformal transformation changes the moduli i.e. $w^{\prime} \rightarrow w^{\prime}+\epsilon$ as we have taken $2 w=1$. As such, we need an $f$ in equation (2.25) such that

$$
f(z)=f(z+2 w), \quad f(z)=2+f\left(z+2 w^{\prime}\right)
$$

Such a function is given by

$$
f(z)=\frac{2 w}{i \pi}\left(\zeta(z)-\frac{\eta}{w} z\right)
$$

Substituting this function with argument $z-z_{i}$ into equation (2.27) we find that

$$
\begin{gathered}
\frac{i \pi}{2 w} \frac{\partial V}{\partial w^{\prime}}=V\left(L_{-2}^{(i)}-\frac{\eta}{w} L_{0}^{(i)}+c\right)+\sum_{j, j \neq i}\left(\zeta\left(z_{j}-z_{i}\right)-\frac{\eta}{w}\left(z_{j}-z_{i}\right)\right) V L_{-1}^{(j)} \\
+\sum_{j, j \neq i}\left(\frac{d \zeta\left(z_{j}-z_{i}\right)}{d z_{j}}-\frac{\eta}{w}\right) V L_{0}^{(j)}+\ldots,
\end{gathered}
$$

where $+\ldots$ means terms with $L_{n}$ for $n \geq 1$. Taking the scalar product of this equation with the vacuum state, with zero momentum, on all string Hilbert spaces and normalizing the vertex as in equation (5.1) we find that

$$
c=\frac{D}{2} N_{11}^{11}=\frac{D}{2}\left(-\frac{\eta}{w}+\frac{\pi i}{2 w\left(w^{\prime}-\bar{w}^{\prime}\right)}\right)
$$

where $D$ is the dimension of space-time and we have used equation (6.1.15)

Applying the zero norm state $\left(L_{-2}^{(i)}+\frac{3}{2}\left(L_{-1}^{(i)}\right)^{2}\right)\left|\Omega^{\prime}\right\rangle_{i}$ on the $i$ th string Hilbert space and arbitrary physical states $\left|\chi_{k}\right\rangle$ to all the other Hilbert spaces to the integrated vertex

$$
W=\int \prod_{j=1}^{N-1} d^{2} z_{j} d^{2} w^{\prime} f_{m} V
$$

we find that

$$
W|\chi\rangle_{(1)} \ldots|\chi\rangle_{(N)}=
$$




$$
\begin{gathered}
=\int \prod_{k=1}^{N-1} d^{2} z_{k} d^{2} w^{\prime} f_{m}\left(\frac{i \pi}{2 w} \frac{\partial V}{\partial w^{\prime}}+V\left(\frac{\eta}{w} L_{0}^{(i)}-c\right)-\sum_{j, j \neq i}\left(\zeta\left(z_{j}-z_{i}\right)-\frac{\eta}{w}\left(z_{j}-z_{i}\right)\right) V L_{-1}^{(j)}\right. \\
\left.-\sum_{j, j \neq i}\left(\frac{d \zeta\left(z_{j}-z_{i}\right)}{d z_{j}}-\frac{\eta}{w}\right) V L_{0}^{(j)}\right)|\chi\rangle_{(1)} \ldots|\Omega\rangle_{(i)} \ldots|\chi\rangle_{(N)} .
\end{gathered}
$$

Using equation (6.2.1) and integrating by parts we find that all the terms containing the $\zeta$ and terms in the same brackets vanish. Integrating by parts we then conclude that

$$
\frac{\partial \ln f_{m}}{\partial \tau}=(D-2) \frac{\eta}{\pi i}-\frac{D}{2} \frac{1}{2\left(w^{\prime}-\bar{w}^{\prime}\right)}
$$

To solve this equation we must recall some facts about theta functions; $\theta_{j}, j=1, \ldots, 4$ are functions of the variable $v$ and also depend on $\tau$, they are sometimes written as $\theta_{j}(v \mid \tau)$. These functions for an arbitrary parameterisation of the torus, i.e. arbitrary $w$ and $w^{\prime}$, obey the equation

$$
\frac{\partial^{2} \theta_{j}(v \mid \tau)}{\partial v^{2}}=4 \pi i \frac{\partial \theta_{j}(v \mid \tau)}{\partial \tau}
$$

Now $\eta$ can be expressed as [24]

$$
\eta=-\frac{1}{12 w} \frac{\left.\frac{\partial^{3} \theta_{1}(v \mid \tau)}{\partial v^{3}}\right|_{v=o}}{\left.\frac{\partial \theta_{1}(v \mid \tau)}{\partial v}\right|_{v=o}}
$$

Using equation (6.2.9) we find that

$$
\eta=-\frac{\pi i}{3 w} \frac{\partial}{\partial \tau}\left(\left.\ln \frac{\partial \theta_{1}(v \mid \tau)}{\partial v}\right|_{v=o}\right)
$$

Examining the explicit expression for $\theta_{1}(v \mid \tau)$ given in the appendix $\mathrm{A}$ we conclude that

$$
\left.\frac{\partial \theta_{1}(v \mid \tau)}{\partial v}\right|_{v=o}=2 \pi q^{\frac{1}{4}}\left(\prod_{n=1}^{\infty}\left(1-q^{2 n}\right)\right)^{3}
$$

where $q=\exp (\pi i \tau)$.

Substituting equation (6.2.11) into equation (6.2.8) and using equation (6.2.12) we find that the measure is given by

$$
f_{m}=\frac{1}{\left|\left(\Pi_{n=1}^{\infty}\left(1-q^{2 n}\right)\right)^{D-2}\right|^{2}} \frac{1}{|q|^{\frac{D-2}{6}}} \frac{1}{(\operatorname{Im} \tau)^{\frac{D}{2}}}
$$

which is indeed the know result. The final contribution containing $\operatorname{Im} \tau$ comes from the last term in equation (6.2.8) arising from the constant in equation (6.2.5), and this in turn 
can be traced to the right hand side of equation (6.1.11), which in turn arises from the $B_{1}$ contour integral in equation (6.1.8). We note that this contribution depends on $\tau$ and $\bar{\tau}$ so one must differentiate with respect to $\tau$ using an expression analogous to that of equation (6.1.18). Hence the non-holomorphic dependence in the measure arises from the use of non-periodic functions in the integrated overlaps.

The modular invariance of the measure requires just such a contribution and one could have taken the $B_{1}$ contour integral to be given by arbitrary expressions times $\alpha_{1}$ and $\alpha_{0}$ oscillators. As is apparent from equation (6.2.5), the former is the one which contributes to the measure through the constant $c$ which first appears in equation (6.2.4). One would then find that the $\alpha_{1}$ term in the $B_{1}$ contour integral takes the value it does in equation (6.1.8). The part of the $B_{1}$ contour integral which contains $\alpha_{0}$ contributes to the nonholomorphic part contained in the purely zero mode contribution of the vertex, and again it is fixed by direct comparison with the known result, or demanding modular invariance.

\subsection{Non-planar one loop open string scattering}

The calculation of one loop open string scattering has many steps in common with that for closed strings given in the previous two sub-sections. The main difference arises from the fact that the open strings sweep out a surface with boundaries and it is on these boundaries that the open strings are emitted, i.e. the Koba-Nielsen points are on the boundaries. At one loop the world-sheet is a cylinder which has two boundaries from which the open strings may be emitted. One can think of the cylinder as being due to an intermediate closed string. The calculation is rather sensitive to how the strings are distributed between the two boundaries. If all the emitted strings are on the same boundary, the world-sheet can be drawn on a plane (the external states being drawn as semi-infinite strips), hence this diagram is called planar. This case has been already computed in $[13,14]$. We want here to calculate the non-planar contribution which has strings on both boundaries (and thus cannot be drawn on a plane). These non-planar contributions are especially relevant to the main subject of this paper as it was shown $[1,4]$ that, at high energy, it is these diagrams that dominate the four tachyon scattering amplitude.

We double the open string world-sheet to construct a torus which, using the Fuchsian representation, is represented as a rectangle in the complex plane, with corners at $z=-w^{\prime}$, $-w^{\prime}+2 w, w^{\prime}+2 w$ and $w^{\prime}$, where $w$ is real and $w^{\prime}$ is purely imaginary. The boundaries of the open string $I$ and $I^{\prime}$ are the segments inside the rectangle with $\operatorname{Im} z=0$ and $\operatorname{Im} z=\operatorname{Im} w^{\prime}$ respectively. We adopt the choices

$$
2 w=-\ln u \quad, \quad w^{\prime}=i \pi
$$

As $w^{\prime}$ and $w$ are purely imaginary and real respectively, the fundamental region is a rectangle for the open string. As $u \leq 1,-\ln u$ is a positive number. In the old literature the quantity $u$ is often called $w$, but following Bateman we are already using $2 w$ for one of the periods of the torus.

We first construct the vertex whose general form is given in equation (5.21). The integrated overlaps with periodic functions, which obey equation (6.1.1), lead to equation (6.1.2) and then equations (6.1.3) and (6.1.4). Thus the coefficients $N_{n m}^{i j}, N_{n}^{i j}, n \geq 2$ in the open string vertex are given by equation (6.1.4). 
As for the closed case there are no periodic functions with a first order pole at a given point on a torus, and we use functions which are shifted by constants when their arguments change by the periods of the torus, i.e. equation (6.1.5). The integrated overlap equation for such functions is given in equation (6.1.6) and consistency then requires us to adopt equation (6.1.7) that is

$$
w^{\prime} \int_{B_{1}} d z V \partial x^{\mu}+w \int_{A_{2}} d z V \partial x^{\mu}=\frac{1}{2} V\left(\sum_{j} z_{j} \alpha_{0}^{\mu(j)}+\sum_{j} \alpha_{1}^{\mu(j)}\right) .
$$

This equation does not determine the individual contour integrals, but the general solutions can be written in the form

$$
\int_{B_{1}} d z V \partial x=b^{(2)} \sum_{j} \alpha_{1}^{(j)}+\sum_{j} b_{j}^{(1)} \alpha_{0}^{(j)}
$$

We will adopt the value

$$
\int_{B_{1}} d z V \partial x=\frac{1}{2} \sum_{j \in I^{\prime}} \alpha_{0}^{(j)}=\frac{1}{2 \pi} \sum_{j} \operatorname{Im}\left(z_{j}-z_{i}\right) \alpha_{0}^{(j)}
$$

This is a natural result; the one loop world-sheet can be seen either as an open string going around the cylinder, or as a closed string propagating from one boundary to the other. In this second picture, the integral on the left-hand side of (6.3.4) measures the momentum of the closed string, which in our case is equal to the total momentum going in the $I^{\prime}$ boundary. The factor of $\frac{1}{2}$ comes from the relation between $p^{\mu}$ and $\alpha_{0}^{\mu}$, which is different for closed and open strings, as can be seen from (5.3) and (5.18). The value of the $B_{1}$ contour integral in equation (6.3.3) feeds into various non-holomorphic corrections to the purely zero mode part of the vertex and the measure as we will explain. While we can not, as for the closed string use modular invariance, we can compare the results with that found long ago for tachyon scattering by sewing tree level three vertices. From this we can deduce the values of $b^{(2)}$ and $b_{j}^{(1)}$. This is in fact the route we have taken, but for simplicity we will use the correct values as given in equation (6.3.4)

Proceeding with the calculation of the vertex, we substitute $\varphi_{1}(z)=\zeta(z)-\frac{\eta}{w} z$ into equation (6.1.6) and use equation (6.3.4) to find

$$
V\left(\alpha_{-1}^{\mu(i)}+\sum_{j} \sum_{m=1}^{\infty} \tilde{N}_{1 m}^{i j} \alpha_{m}^{\mu(j)}+\sum_{j} \tilde{N}_{1}^{i j} \alpha_{0}^{\mu(j)}\right)=-\frac{i}{2 w} V \sum_{j} \operatorname{Im}\left(z_{j}-z_{i}\right) \alpha_{0}^{(j)}
$$

where the $\tilde{N}$ 's are given in equation (6.1.13). The coefficients in the vertex as calculated from equation (6.3.5) are given by

$$
N_{1}^{i j}=\tilde{N}_{1}^{i j}+\frac{i}{2 w} \operatorname{Im}\left(z_{j}-z_{i}\right), \quad \tilde{N}_{1 m}^{i j}=N_{1 m}^{i j} .
$$


We note that we only get a contribution from the non-periodicity of $\varphi_{1}$ when $z_{j}$ and $z_{i}$ are on different boundaries.

We assumed in the derivation of the purely zero mode piece that the coefficients in the vertex are the derivatives of a single function. We now show that this is indeed the case for the above vertex. In doing so we will treat the cases of whether $z_{j}$ and $z_{i}$ are on the same boundary or not differently. If they are on the same boundary, the non-periodic nature of $\varphi_{1}$ has no effect and so $\operatorname{Im}\left(z_{j}-z_{i}\right)=0$ and $N_{1}^{i j}=\tilde{N}_{1}^{i j}$. As the reader will readily verify, for these $i$ and $j$ all the coefficients are given by equations (5.23) and (5.26) if we take

$$
\ln \psi(z)=\ln \sigma(z)-\frac{\eta}{2 w} z^{2}-2 \ln w=\ln \frac{\theta_{1}(v)}{\theta_{1}^{\prime}(0)},
$$

where $v=\frac{z}{2 w}$. The zero mode coefficient is then given by equation (5.24). These are the same as for the case of the planar string.

If $z_{i}$ and $z_{j}$ are on different boundaries, we have $\operatorname{Im}\left(z_{j}-z_{i}\right)= \pm \pi$ and so from equation (6.3.6) we have $N_{1}^{i j}=\tilde{N}_{1}^{i j} \pm \frac{i \pi}{2 w}$. However, in this case, two of the Koba-Nielsen points are on the line $\operatorname{Im} z=0$ while the other two are on the line $\operatorname{Im} z=\pi$ within the fundamental region. As it is desirable to integrate over the real variable $\operatorname{Re} z_{j}$ we will consider functions of $\operatorname{Re} z_{j}$ and absorb the imaginary contributions to the Koba-Nielsen points in the definition of the function. Taking these two requirements into account, the function that leads to the coefficients of the vertex using equations (5.23) and (5.26) is $\psi_{T}(z)$, which is given by

$$
\ln \psi_{T}=\ln \frac{\theta_{4}(\operatorname{Re} v)}{\theta_{1}^{\prime}(0)}
$$

To see that this is the case we need the identity [24]

$$
\ln \frac{\theta_{4}(z)}{\theta_{4}(0)}=\ln \sigma\left(z+w^{\prime}\right)-\eta^{\prime} z-\frac{\eta}{2 w} z^{2}-\ln \sigma\left(w^{\prime}\right)
$$

we recall that $v=\frac{z}{2 w}$. Differentiating equation (6.3.8) and using the above identity we find that

$$
\begin{gathered}
\left.\frac{d}{d t} \ln \psi_{T}\left(\operatorname{Re} z_{j}-\operatorname{Re} z_{i}+t\right)\right|_{t=0}=\zeta\left(\operatorname{Re} z_{j}-\operatorname{Re} z_{i}+i \pi\right)-\eta^{\prime}-\frac{\eta}{w}\left(\operatorname{Re} z_{j}-\operatorname{Re} z_{i}\right) \\
=\zeta\left(\operatorname{Re} z_{j}-\operatorname{Re} z_{i} \pm i \pi\right)-\frac{\eta}{w}\left(\operatorname{Re} z_{j}-\operatorname{Re} z_{i} \pm i \pi\right) \pm \frac{i \pi}{2 w}
\end{gathered}
$$

where in deriving the last equation we have used the Legendre relation and $\zeta\left(z+2 w^{\prime}\right)=$ $\zeta(z)+2 \eta^{\prime}$ to derive the relationship with the lower sign. Hence, we find that

$$
\left.\frac{d}{d t} \ln \theta_{4}\left(\operatorname{Re} v_{j}-\operatorname{Re} v_{i}+t\right)\right|_{t=0}=\varphi_{1}\left(\operatorname{Re} z_{j}-\operatorname{Re} z_{i} \pm i \pi\right) \pm \frac{i \pi}{2 w}
$$

In the argument of $\varphi_{1}$ we recognise the positions of the Koba-Nielsen points as they are on the rectangle, i.e we find in this equation $\varphi_{1}\left(z_{j}-z_{i}\right)$ and the last term is just that 
required to get the correct coefficient $N_{1}^{i j}$ in equation (6.3.6). This last term disappears when taking further derivatives and one recovers all the other coefficients in the vertex given above using equations (5.23) and (5.26).

In summary, we have

$$
N^{i j}=\left\{\begin{array}{c}
\ln \psi\left(z_{j}-z_{i}\right) \quad i, j \text { on the same boundary } \\
\ln \psi_{T}\left(\operatorname{Re} z_{j}-\operatorname{Re} z_{i}\right) \quad i, j \text { on different boundaries }
\end{array}\right.
$$

with

$$
\begin{array}{r}
\ln \psi(z)=\ln \frac{\theta_{1}(v)}{\theta_{1}^{\prime}(0)} \\
\ln \psi_{T}(z)=\ln \frac{\theta_{4}(v)}{\theta_{1}^{\prime}(0)}
\end{array}
$$

where $\theta_{j}^{\prime}(0)=\left.\frac{\partial \theta_{j}(v \mid \tau)}{\partial v}\right|_{v=0}$. We note that if $i, j$ are on the same boundary $z_{j}-z_{i}$ is real.

The calculation of the measure for the open string has many steps in common with that for the closed string given in section 6.2. From the decoupling of $L_{-1}^{(j)}|\Omega\rangle$, we conclude that the measure $f_{m}$ is independent of $z_{j}$. For the decoupling of the zero norm physical states at higher levels, we must understand how to change to dependence of the vertex on its modulus using a conformal transformation while $w^{\prime}=i \pi$ is fixed and we change $u \rightarrow u(1-\epsilon)$. Under this change $w \rightarrow w+\frac{\epsilon}{2}$ which is implemented by the conformal transformation with an $f$ in equation $(2.25)$ such that

$$
f(z)=f\left(z+2 w^{\prime}\right), \quad f(z)=1+f(z+2 w) .
$$

Such a function is given by

$$
f(z)=-\left(\zeta(z)-\frac{\eta^{\prime}}{i \pi} z\right)
$$

Substituting this function with argument $z-z_{i}$ into equation (2.27) we find that

$$
\begin{gathered}
u \frac{\partial V}{\partial u}=V\left(L_{-2}^{(i)}-\frac{\eta^{\prime}}{w^{\prime}} L_{0}^{(i)}+c\right)+\sum_{j, j \neq i}\left(\zeta\left(z_{j}-z_{i}\right)-\frac{\eta^{\prime}}{w^{\prime}}\left(z_{j}-z_{i}\right)\right) V L_{-1}^{(j)} \\
+\sum_{j, j \neq i}\left(\frac{d \zeta\left(z_{j}-z_{i}\right)}{d z_{j}}-\frac{\eta^{\prime}}{w^{\prime}}\right) V L_{0}^{(j)}+\ldots
\end{gathered}
$$

Taking the scalar product of this equation with the vacuum state, with zero momentum, on all string Hilbert spaces and normalizing the vertex as in equation (5.21) we find that

$$
c=\frac{D}{2} N_{11}^{11}=-\frac{D}{2} \frac{\eta}{w} \text {. }
$$

Applying the zero norm state $\left(L_{-2}^{(i)}+\frac{3}{2}\left(L_{-1}^{(i)}\right)^{2}\right)\left|\Omega^{\prime}\right\rangle_{i}$ on the $i$ th string Hilbert space and arbitrary physical states $\left|\chi_{k}\right\rangle$ to all the other Hilbert spaces to the integrated vertex, we find that

$$
\int \prod_{k=1}^{N-1} d z_{k} d u\left(f_{m}\left(u \frac{\partial V}{\partial u}+V \frac{\eta^{\prime}}{w^{\prime}} L_{0}^{(i)}-V c\right)-\sum_{j, j \neq i}\left(\zeta\left(z_{j}-z_{i}\right)-\frac{\eta^{\prime}}{w^{\prime}}\left(z_{j}-z_{i}\right)\right) V L_{-1}^{(j)}\right.
$$




$$
\left.-\sum_{j, j \neq i}\left(\frac{d \zeta\left(z_{j}-z_{i}\right)}{d z_{j}}-\frac{\eta^{\prime}}{w^{\prime}}\right) V L_{0}^{(j)}\right)|\chi\rangle_{(1)} \ldots|\Omega\rangle_{(i)} \ldots|\chi\rangle_{(N)}=0 .
$$

As for the closed string, the terms containing the $\zeta$ and other terms in the same brackets vanish by integration by parts. Integrating by parts with respect to $u$, and using Legendre's relation we then conclude that

$$
u \frac{\partial \ln f_{m}}{\partial u}=\frac{(D-2)}{2} \frac{\eta}{w}+\frac{1}{2 w}-1
$$

To solve this equation we require an expression for $\eta$ in terms of $u$. Using the well known relation

$$
\left.\frac{\partial \theta_{1}\left(v \mid \frac{a \tau+b}{c \tau+d}\right)}{\partial v}\right|_{v=0}=\left.e(c \tau+d)^{\frac{3}{2}} \frac{\partial \theta_{1}(v \mid \tau)}{\partial v}\right|_{v=0}
$$

where $e$ is a constant whose value will not be important in what follows, and equation (6.2.12) we find that

$$
q^{\frac{1}{12}} \prod_{n=1}^{\infty}\left(1-q^{2 n}\right)=u^{\frac{1}{24}}\left(\frac{-\ln u}{2 \pi}\right)^{\frac{1}{2}} \prod_{n=1}^{\infty}\left(1-u^{n}\right)
$$

Using equation (6.2.11) we then find that the measure for one loop non-planar scattering is given by

$$
f_{m}=\frac{1}{u u^{\frac{D-2}{24}}\left(\prod_{n=1}^{\infty}\left(1-u^{n}\right)\right)^{D-2}(-\ln u)^{D / 2}} .
$$

This displays the expected reduction in degrees of freedom from $D$ to $D-2$.

\section{Scattering at Higher Genus and Discussion}

It was argued in references $[2,3,4]$ that the four tachyon closed string scattering is dominated, at high energy and fixed angle, by a world-sheet surface of the form

$$
y^{N}=\frac{\left(\varsigma-\varsigma_{1}\right)\left(\varsigma-\varsigma_{2}\right)}{\left(\varsigma-\varsigma_{3}\right)\left(\varsigma-\varsigma_{4}\right)} \equiv(Q(\varsigma))^{N}
$$

This is a Riemann sphere with two branch cuts of degree $N$, and so is in effect a set of $N$ Riemann spheres connected by the two branch cuts which are at the same points for each sheet. It is a surface of genus $g=N-1$. The strings are emitted from the points at the beginning and end of the branch cuts, i.e. the Koba-Nielsen points are $\varsigma_{i}, i=1,2,3,4$. The authors of references $[2,3,4]$ argued that the tachyon scattering contains an exponential factor, bilinear in the momenta, which is $\frac{1}{N}$ times the tree level result. This essentially results from the fact that the Green's function is identical on all the sheets and there are $N$ of them.

We will now use the group theoretic method to derive some of the string vertex for the four closed string scattering at arbitrary genus, and we will conjecture its complete form. 
The meromorphic functions which are well defined on the surface specified by equation (7.1) are rational polynomials in $Q$ and $\varsigma$. We are interested in functions that have poles at one of the Koba-Nielsen points. Clearly, one set of such functions is

$$
\frac{1}{\left(\varsigma-\varsigma_{i}\right)^{p}}=\frac{1}{\left(\varrho_{i}\right)^{N p}}
$$

where $\varrho_{i}=\left(\varsigma-\varsigma_{i}\right)^{\frac{1}{N}}$. However, one can also consider

$$
\frac{Q^{r}\left(\varsigma-\varsigma_{4}\right)}{\left(\varsigma-\varsigma_{3}\right)^{p}}=\frac{a_{0}^{(3)}}{\left(\varrho_{3}\right)^{N p+r}}+\frac{a_{1}^{(3)}}{\left(\varrho_{3}\right)^{N(p-1)+r}}+\ldots \quad, \quad 1 \leq r<N, p \geq 1
$$

where $+\ldots$ indicates less singular terms, and a similar expression with $\varsigma_{3}$ and $\varsigma_{4}$ exchanged for the pole at $\varsigma_{4}$. For poles at $\varsigma_{1}$ we take the functions

$$
\frac{\left(\varsigma-\varsigma_{2}\right)}{Q^{r}\left(\varsigma-\varsigma_{1}\right)^{p}}=\frac{a_{0}^{(1)}}{\left(\varrho_{1}\right)^{N p+r}}+\frac{a_{1}^{(1)}}{\left(\varrho_{1}\right)^{N(p-1)+r}}+\ldots \quad, \quad 1 \leq r<N, p \geq 1
$$

with an analogous expression for the poles at $\varsigma_{2}$. We note that the functions in equations (7.3) and (7.4) are of the form $\varrho_{i}^{-(N p+r)}$ times a polynomial in $\varrho_{i}^{N}$ and, in particular, they contain no constant term. Furthermore these functions vanish at the Koba-Nielsen points where they are not singular. We will need a good set of local coordinates in the neighbourhood of each of the Koba-Nielsen points, and one such choice is $\varrho_{i}$. We note that $\varsigma-\varsigma_{i}$ is not a good choice for reasons spelt out in the genus one case. Equations (7.2) and (7.3) have all possible poles at a single Koba-Nielsen point except for $\frac{1}{\left(\varrho_{i}\right)^{q}}, q=1, \ldots, N-1$. These are the expected missing poles for which no such function exists.

Using the functions of equation (7.2) in the integrated overlap equation (2.15) we conclude that, in the high energy limit,

$$
V_{\varrho}\left(\alpha_{-N p}^{\mu(i)}+\sum_{j, j \neq i} \frac{1}{\left(\varsigma_{j}-\varsigma_{i}\right)^{p}} \alpha_{0}^{\mu(j)}\right)=0
$$

Using superpositions of the functions in equations (7.3) and (7.4) we also find, in the high energy limit, integrated overlap equations of the form

$$
V_{\varrho}\left(\alpha_{-(N p+r)}^{\mu(i)}+c_{i} \alpha_{-r}^{\mu(i)}\right)=0 \quad, \quad r=1, \ldots, N-1, p \geq 1
$$

where $c_{i}$ is a constant. However, to find the dependence of the vertex on $\alpha_{q}^{\mu}, 1 \leq q<N$ we must use integrated overlap equations derived from functions $\varphi$ that shift by a constant under the periods of the surface.

The dependence of the vertex on the oscillators $\alpha_{N p}^{\mu}, \bar{\alpha}_{N p}^{\mu}, p \geq 1$ and $\alpha_{0}^{\mu}$ is given by

$$
\prod_{k=1}^{N}{ }_{(k)}\langle 0| \exp \left(-\sum_{i, j} \sum_{p=1}^{\infty}\left(\alpha_{p N}^{\mu(i)} \frac{1}{p N\left(\varsigma_{j}-\varsigma_{i}\right)^{p}} \alpha_{0 \mu}^{(j)}+\bar{\alpha}_{p N}^{\mu(i)} \frac{1}{p N\left(\overline{\varsigma_{j}}-\overline{\varsigma_{i}}\right)^{p}} \alpha_{0 \mu}^{(j)}\right)\right.
$$




$$
\left.+\frac{1}{N} \sum_{i, j} \alpha_{0}^{\mu(i)} \ln \left|\left(\varsigma_{j}-\varsigma_{i}\right)\right| \alpha_{0 \mu}^{(j)}\right) .
$$

The purely momentum piece is that derived in references $[2,3,4]$.

We recall from section four that the genus one vertex does not depend on $\alpha_{1}^{\mu}$ as a consequence of the overlap relations derived from a non-periodic function $\varphi$. The integrated overlap equations which are constructed from periodic functions whose first terms have odd negatively moded oscillators then imply that the vertex then does not depend on $\alpha_{2 n+1}^{\mu}, n=1,2, \ldots$. The generalisation of the first of these statement to higher genus is that the vertex does not depend on the oscillators whose dependence is determined by the overlap identities which require non-periodic functions, that is those constructed from functions $\varphi$ associated with the missing poles on the Riemann surface and so it does not depend on $\alpha_{q}^{\mu} q=1, \ldots, N-1$. If this is true then the overlap equations (7.6) then implies that the vertex does not depend on the oscillators $\alpha_{p N+r}^{\mu}, r=1, \ldots, N-1, p \geq 1$. In other words it depends on only $\alpha_{p N}^{\mu}, p \geq 1$ and as a result the vertex in equation (7.7) is the complete vertex for the scattering of four high energy, fixed angle, arbitrary strings.

To verify this assumption one has to find the contour integrals of $\int V \partial x^{\mu} d z$ around the cuts in the surface. We will leave this to a later paper. However, the vertex must satisfy the overlap equation $V x^{\mu(i)}\left(\varrho_{i}\right)=V x^{\mu(j)}\left(\varrho_{j}\right)$. It is easy to verify that the vertex of equation (7.7) does satisfy this equation. Thus if there was some additional oscillator dependence in the vertex this factor would have to satisfy this equation by itself; perhaps this is unlikely as all other vertices encountered so far in string theory do not work in this way. In the next section we will give a path integral derivation of this vertex.

Although the vertex does not satisfy any genus independent relations, it does have a very simple form. As one goes to higher and higher genus, the scattering becomes free-like as more and more of the coefficients in the vertex become zero. Indeed, for the contributions calculated in this paper, a process involving a graviton as one of the scattered particles receives only a tree contribution as all higher genus vertices do not contain the required $\alpha_{1}$ oscillator. Indeed, if a state contains an oscillator $\alpha_{n}$ this can only get contributions from genus g where $n=p(g+1)$ for positive integer $p$. As a result, the contribution to the scattering of states involving oscillators $\alpha_{n_{i}}^{\mu(i)}$ occurs at most at the greatest common divisor of the $n_{i}$ minus one. What is also striking is that the coefficients that are nonzero are essentially tree-like. In this paper we have calculated the leading term in the high energy limit at each genus. This is a term of the form of the tachyon contribution multiplied by $p^{m}$ where $m$ is the number of oscillators in all the four particle physical states which results from the $\alpha_{n} \alpha_{0}$ term in the exponential of the vertex. However, this is not the same as the dominant contribution taking into account the different orders of perturbation theory. Indeed, at one loop there is a contribution from the full vertex which arises from the $\alpha_{n} \alpha_{m}$ term in the exponential of the vertex. Although this involves no momenta, it is larger than the tree level contribution referred to above because the exponential factor at one loop is much larger than that found at tree level.

It will be interesting to see if the results found in this paper can be used to find any symmetries hidden in the high energy scattering amplitudes. We have in mind the symmetries proposed in reference [7] and also those associated with higher spin theories. 
It will also be of interest to generalise these results to the superstring. One expects that at least the Neveu-Schwarz sector of the calculation would be rather similar. However, in this theory the tachyon is projected out in the superstring and so for the particles that are actually in the theory it will be interesting to find the analogous high energy scattering.

\section{Derivation of Four Arbitrary String Scattering from the Sum over World Sheets Approach.}

In this section we recover the results for four arbitrary closed string scattering at high energy from the sum over world sheets approach to string theory used in references [2-5]. In this approach the amplitude is given by a Euclidean path integral of the form

$$
A=\int \mathcal{D} g_{\alpha \beta} \mathcal{D} x^{\mu} \exp \left(-S\left[x^{\mu}\right]\right) \prod_{i} \int d^{2} \xi_{i} V^{(i)}\left(x^{\mu}\left(\xi_{i}\right), p_{i}\right) .
$$

In this equation

$$
S\left[x^{\mu}\right]=\frac{1}{4 \pi \alpha^{\prime}} \int d^{2} \xi \sqrt{g} g^{\alpha \beta} \partial_{\alpha} x^{\mu} \partial_{\beta} x_{\mu}=\int d^{2} \xi \frac{1}{2} x^{\mu}(\xi) P x_{\mu}(\xi)
$$

where $P$ is the operator $P=-\frac{1}{2 \pi \alpha^{\prime}} \partial_{\alpha}\left(\sqrt{g} g^{\alpha \beta} \partial_{\beta}\right)$, and $V^{(i)}\left(x^{\mu}\left(\xi_{i}\right), \partial x^{\mu}\left(\xi_{i}\right), \ldots, p_{i}\right)$ is the vertex operator of conformal weight $(1,1)$ corresponding to the emitted string at the Koba-

Nielsen point $z_{i}$ with momentum $p_{i}=\sqrt{\frac{2}{\alpha^{\prime}}} \alpha_{0}=\sqrt{\frac{2}{\alpha^{\prime}}} \bar{\alpha}_{0}$. The traditional choice of $\alpha^{\prime}$ is $\alpha^{\prime}=2$ for the closed string, although we will not make this choice in this paper. The vertex operator is of the form

$$
\begin{gathered}
V^{(i)}\left(x^{\mu}\left(\xi_{i}\right), \partial x^{\mu}\left(\xi_{i}\right), \ldots, p_{i}\right)=\sqrt{g}\left(\epsilon_{\mu \nu} \partial^{p} x^{\mu}\left(\xi_{i}\right) \bar{\partial}^{p} x^{\nu}\left(\xi_{i}\right)+\ldots\right) \exp \left(i p_{i} \cdot x\left(\xi_{i}\right)\right) \\
\equiv U\left(x^{\mu}\left(\xi_{i}\right), \partial x^{\mu}\left(\xi_{i}\right), \ldots\right) \exp \left(i p_{i} \cdot x\left(\xi_{i}\right)\right)
\end{gathered}
$$

where $+\ldots$ in the first line stands for the addition of terms which contain more than two factors of $x^{\mu}$ which are also compatible with the level matching condition of the closed string. In this expression $\epsilon_{\mu \nu}$ is one of the polarisation tensors needed to specify the physical state. Demanding that the vertex operator be of conformal weight $(1,1)$ implies that the polarisation tensors are subject to certain conditions.

The path integral is over all surfaces of the genus being considered. As we are working in the critical dimension, namely twenty-six, the integral is invariant under the Weyl rescalings of the metric $g_{\alpha \beta}$ and diffeomorphisms and so these must be factored out of the path integral by incorporating ghosts. The integral is then over the remaining variables; the moduli of the Riemann surfaces $[31,34]$ of genus $g$, which is a finite dimensional space, and the Koba-Nielsen coordinates $z_{i}$.

We may write the path integral of equation (8.1) as

$$
A=\int \mathcal{D} g_{\alpha \beta} \Pi_{l} d^{2} \xi_{l} \mathcal{D} x^{\mu} \exp \left(-S\left[x^{\mu}\right]+\int d^{2} \xi \sum_{j} i p_{j} \cdot x(\xi) \delta\left(\xi-\xi_{j}\right)\right)
$$




$$
\prod_{k} U^{(k)}\left(x^{\mu}\left(\xi_{k}\right), \partial x^{\mu}\left(\xi_{k}\right), \ldots\right)
$$

Completing the square in $x^{\mu}$ in the first exponential this expression becomes

$$
\begin{gathered}
A=\int \mathcal{D} g_{\alpha \beta} \Pi_{l} d^{2} \xi_{l} \mathcal{D} \tilde{x}^{\mu} \exp \left(-S\left[\tilde{x}^{\mu}\right]\right) \exp \left(-\frac{1}{2} \sum_{j, k} p_{j} \cdot G\left(\xi_{j}, \xi_{k}\right) p_{k}\right) \\
\prod_{k} U^{(k)}\left(x^{\mu}\left(\xi_{k}\right), \partial x^{\mu}\left(\xi_{k}\right), \ldots\right)
\end{gathered}
$$

where $G\left(\xi_{j}, \xi_{k}\right)$ is the Green's function on the surface which is defined to satisfy the equation

$$
P G\left(\xi, \xi_{k}\right)=-\frac{1}{2 \pi \alpha^{\prime}} \partial_{\alpha}\left(\sqrt{g} g^{\alpha \beta} \partial_{\beta}\right) G\left(\xi, \xi_{k}\right)=\delta\left(\xi, \xi_{k}\right)
$$

and

$$
\tilde{x}^{\mu}(\xi)=x^{\mu}(\xi)-i \sum_{j} p_{j}^{\mu} P^{-1} \delta\left(\xi, \xi_{j}\right)=x^{\mu}(\xi)-i \sum_{j} p_{j}^{\mu} G\left(\xi, \xi_{j}\right) .
$$

The Green's function $G$ depends on the moduli of the Riemann surface.

In the high energy limit we may replace the dependence of $U^{(i)}$, in equation (8.5), on $x^{\mu}\left(\xi_{i}\right)$ by $\hat{x}^{\mu}\left(\xi_{i}\right)$ where

$$
\hat{x}^{\mu}(\xi)=i \sum_{j} p_{j}^{\mu} G\left(\xi, \xi_{j}\right) .
$$

We may now carry out the integration over $\tilde{x}^{\mu}$ to produce the usual determinants and, following references [2-5], the high energy limit can now be calculated by the method of steepest descent by extremising in the moduli of the Riemann surface and the positions of the Koba-Nielsen points $\xi_{i}$. The values of these parameters are determined by the second exponential factor in equation (8.5) involving the momenta and the Green's function. As such, we find that the amplitude for string scattering is given by that for the scattering of four tachyons, $A_{\text {tachyon }}$ multiplied by the $U$ factors associated with the scattering of the arbitrary string states, that is

$$
A=A_{\text {tachyon }} \prod_{k} U^{(k)}\left(\hat{x}^{\mu}\left(\hat{\xi}_{k}\right), \partial \hat{x}^{\mu}\left(\hat{\xi}_{k}\right), \ldots\right)
$$

where $\hat{\xi}_{k}$ are the values of the Koba-Nielsen points at the extremum. The values of the moduli and Koba-Nielsen points at the extremum fix the world-sheet to be the $\hat{x}^{\mu}$ given in equation (8.8)

It was argued in references [2-5] that in the high energy limit the dominant Riemann surface at genus $g$ for four particle scattering could be represented by the Riemann sphere with two branch cuts of degree $N=g+1$ and the four Koba-Nielsen points were at the positions $z_{i}$ of the branch cuts. As such, the surface consisted of a $N$-sheeted cover of the Riemann sphere given by

$$
y^{N}=\frac{\left(\varsigma-\varsigma_{1}\right)\left(\varsigma-\varsigma_{2}\right)}{\left(\varsigma-\varsigma_{3}\right)\left(\varsigma-\varsigma_{4}\right)}
$$


Since the sheets are all on an equal footing the Green's function on the surface is given by $[2-5]$

$$
G\left(\varsigma, \varsigma^{\prime}\right)=-\frac{\alpha^{\prime}}{2 N} \ln \left(\varsigma-\varsigma^{\prime}\right)+\text { c.c. }
$$

Using this result we can find an explicit expression for the extremal world surface given in equation (8.8). However, it is important to remember that $\varsigma-\varsigma_{i}$ is not a good coordinate at the position of the Koba-Nielsen point $\varsigma_{i}$ where the string is emitted. A good coordinate is given by $\varrho_{i}=\left(\varsigma-\varsigma_{i}\right)^{\frac{1}{N}}$. The string surface evaluated in terms of the good coordinate $\varrho_{i}$ is given by

$$
\hat{x}^{\mu}\left(\varrho_{i}\right)=-\frac{i \alpha^{\prime}}{2} p_{i}^{\mu} \ln \varrho_{i}-\frac{i \alpha^{\prime}}{2 N} \sum_{j, j \neq i} p_{j}^{\mu} \ln \left(\varrho_{i}^{N}+\varsigma_{i}-\varsigma_{j}\right)+c . c
$$

Taking the derivative and expanding about $\varrho_{i}=0$ we find that

$$
\begin{gathered}
\partial \hat{x}^{\mu}\left(\varrho_{i}\right)=-\frac{i \alpha^{\prime}}{2 \varrho_{i}} p_{i}^{\mu}-\frac{i \alpha^{\prime}}{2} \sum_{j, j \neq i} p_{j}^{\mu} \frac{\varrho_{i}^{N-1}}{\left(\varrho_{i}^{N}+\varsigma_{i}-\varsigma_{j}\right)}+c . c \\
=-\frac{i \alpha^{\prime}}{2 \varrho_{i}} p_{i}^{\mu}+\frac{i \alpha^{\prime}}{2} \sum_{j, j \neq i} \sum_{p=0}^{\infty} p_{j}^{\mu} \frac{\varrho_{i}^{N(p+1)-1}}{\left(\varsigma_{j}-\varsigma_{i}\right)^{p+1}}+c . c
\end{gathered}
$$

Recalling the standard expression for the embedding field in terms of oscillators, $\partial x^{\mu}(\varrho)=$ $-i \sqrt{\frac{\alpha^{\prime}}{2}} \sum_{-\infty}^{\infty} \alpha_{-n}^{\mu} \varrho^{n-1}$, we conclude that in the high energy limit the oscillators are given by

$$
\hat{\alpha}_{-N p}^{\mu(i)}=-\sum_{j, j \neq i} \frac{\hat{\alpha}_{0}^{\mu(j)}}{\left(\varsigma_{j}-\varsigma_{i}\right)^{p}}, p=0,1, \ldots \text { and } \hat{\alpha}_{0}^{\mu(i)}=\sqrt{\frac{\alpha^{\prime}}{2}} p_{i}^{\mu}
$$

with all other oscillators vanishing. We note that had we not used the good coordinate $\varrho_{i}$ we would not have recovered the correct dependence of $\hat{x}^{\mu}\left(\varrho_{i}\right)$ on the momentum.

Given an operator of conformal weight $(1,1)$ of the form of that in equation (8.3) we can construct the associated physical state in the usual way

$$
\lim _{\varrho_{i} \rightarrow 0} V^{(i)}\left(x^{\mu}\left(\varrho_{i}\right), p_{i}\right)|0\rangle=\left|\psi_{i}\right\rangle
$$

The positively moded oscillators, $\alpha_{m}, m>0$, in $V^{(i)}\left(x^{\mu}\left(\varrho_{i}\right), p_{i}\right)$ annihilate on the vacuum, while those that have $\varrho_{i}^{n}, n>0$ also vanish in the limit and one is left with a finite number of negatively moded oscilators acting on the vacuum which now carries the momentum of the string state. We note that some potentially divergent terms as $\varrho_{i} \rightarrow 0$ are absent due to the physical state conditions, or equivalently, the demand that the operator is of conformal weight $(1,1)$ satisfied by the polarisation tensors.

The arbitrary four string amplitude in equation (8.9) requires us to evaluate the conformal operator at the Koba-Nielsen point $\varsigma_{i}$, or $\varrho_{i}=0$. This means evaluating the expression of equation (8.3) for $\partial^{p} \hat{x}^{\mu}\left(\varrho_{i}\right)$ at $\varrho_{i}=0$. However, we see from equation (8.13) 
that this field contains no positively moded oscillators and is analytic in $\varrho_{i}$, apart from some terms containing momentum that vanish in the vertex operator due to the physical state conditions among the polarisation tensors. Hence, it has a smooth limit when $\varrho_{i} \rightarrow 0$ which is precisely the same as the expression of equation (8.15) for the physical state, except that the oscillators acting on the vacuum in that equation are replaced by the hatted oscillators which are given in terms of the momenta by equation (8.14). On the other hand, when evaluating the scattering amplitude using the group theoretic approach we apply the desired physical state onto the vertex and then evaluate it by acting with the negatively moded oscillators to the left, that is onto the vertex. In doing so we bring down certain factors from the vertex which in the high energy limit contain just the momenta. We observe that for the vertex of equation (7.7) these are just those given in equation (8.14), see equation (7.5). As a result, we find that the sum over world surfaces approach recovers the four arbitrary string scattering of the group theoretic approach embodied in the vertex of equation (7.7).

The ease with which one can recover the result for four arbitrary string scattering from the sum over world surfaces approach makes up for some of the heuristic steps that are required, such as the precise definition of the vertex operators used. Nonetheless it does provide strong confirmation for the vertex of equation (7.7).

\section{Acknowledgments}

This research was supported by a PPARC senior fellowship PPA/Y/S/2002/001/44 and by the PPARC grants PPA/G/O/2002/00475. N. M. would like to thank the Theory Group at CERN for their kind hospitality during the writing up of this project.

\section{Appendix A: Representations of genus one Riemann surfaces}

In this Appendix, we carefully review different representations of the torus, namely the parallelogram and the cut sphere representations, and how they are related. We give a short review of the theory of elliptic functions, the Weierstrass function $\mathcal{P}(z)$, the zeta function $\zeta(z)$ and the sigma function $\sigma(z)$, and their relations to the Jacobi theta functions. For more details, we refer the reader to [24] for example.

A torus can be mapped conformally to a parallelogram in the complex plane, whose opposite sides are identified. Such a parallelogram can be obtained by modding out the complex plane $\mathbf{C}$ by the equivalence relations.

$$
z \sim z+2 m w+2 n w^{\prime} \quad m, n \in \mathbf{Z} .
$$

The parallelogram in $\mathbf{C}$ with corners at $z=0,2 w, 2 w+2 w^{\prime}$ and $2 w^{\prime}$ is mapped in ane to one manner onto the torus. We call the region inside the parallelogram the fundamental domain. We will assume that $w$ and $w^{\prime}$ are labelled in such a way that $\operatorname{Im} \tau>0$, where

$$
\tau=\frac{w^{\prime}}{w}
$$

Of fundamental importance are the holomorphic maps from the torus onto the Riemann sphere. These can be seen as maps $f$ from the complex numbers to the Riemann sphere, which have to obey the periodicity conditions:

$$
f(z+2 w)=f(z) \quad, \quad f\left(z+2 w^{\prime}\right)=f(z) \quad \forall z \in \mathbf{C} .
$$


Since we want $f$ to be onto, it must have at least one pole (because the Riemann sphere is $\mathbf{C} \cup\{\infty\}$ ). However one can see that $f$ cannot have only one pole; indeed if we integrate $f$ along the circumference of the fundamental parallelogram, we will get zero because the contributions from opposite sides will cancel. Therefore the sum of the residues of $f$ inside the fundamental region is zero. But $f$ can have two or more poles, or multiple poles. In fact all such functions can be constructed from two functions: the Weierstrass $\mathcal{P}$ function and its derivative $\mathcal{P}^{\prime}(z)$. The Weierstrass function has one double pole at $z=0$ and is holomorphic everywhere else. It is given by

$$
\mathcal{P}(z)=\mathcal{P}\left(z \mid w, w^{\prime}\right)=\frac{1}{z^{2}}+\sum^{\prime}\left(\frac{1}{\left(z-2 m w-2 n w^{\prime}\right)^{2}}-\frac{1}{\left(2 m w+2 n w^{\prime}\right)^{2}}\right)
$$

where the prime on the sum indicates that we exclude the point $(m, n)=(0,0)$ from the summation. This function is even and it is normalized so that the coefficient of the leading term in its power expansion is one, and the constant term is zero. Its series expansion thus starts with

$$
\mathcal{P}(z)=\frac{1}{z^{2}}+\frac{g_{2}}{20} z^{2}+\frac{g_{3}}{28} z^{4}+\ldots
$$

where $g_{2}$ and $g_{3}$ are given by

$$
g_{2}=60 \sum^{\prime}\left(2 m w+2 n w^{\prime}\right)^{-4} \quad, \quad g_{3}=140 \sum^{\prime}\left(2 m w+2 n w^{\prime}\right)^{-6} .
$$

The derivative of the $\mathcal{P}$ function, $\mathcal{P}^{\prime}(z)$, satisfies the elliptic condition (A.3) and is odd. It is then easy to see that

$$
\mathcal{P}^{\prime}(w)=\mathcal{P}^{\prime}\left(w^{\prime}\right)=\mathcal{P}^{\prime}\left(w+w^{\prime}\right)=0
$$

At these special points, it is useful to define

$$
\varsigma_{1}=\mathcal{P}(w) \quad, \quad \varsigma_{2}=\mathcal{P}\left(w+w^{\prime}\right) \quad, \quad \varsigma_{3}=\mathcal{P}\left(w^{\prime}\right)
$$

Using this, (A.7) can be written

$$
\mathcal{P}^{\prime}\left(\varsigma_{i}\right)=0 \quad i=1,2,3
$$

One of the fundamental properties of $\mathcal{P}(z)$ is that its derivative satisfies the algebraic equation

$$
\mathcal{P}^{\prime 2}(z)=4 \mathcal{P}^{3}(z)-g_{2} \mathcal{P}(z)-g_{3}=4\left(\mathcal{P}(z)-\varsigma_{1}\right)\left(\mathcal{P}(z)-\varsigma_{2}\right)\left(\mathcal{P}(z)-\varsigma_{3}\right)
$$

where, in the last equality, we have used (A.9). A way to see why we have such a relation is to note that both sides of this equation have the same principal part (the part of the series expansion with negative powers), their difference is therefore a holomorphic function from the torus to the sphere. It maps the torus to a set that is compact and doesn't contain infinity, hence bounded. From Liouville's theorem, we deduce that this function 
is a constant. We may then use (A.4) to show that this constant is zero. It follows from (A.10) that

$$
\mathcal{P}^{\prime \prime}(z)=6 \mathcal{P}^{2}(z)-\frac{1}{2} g_{2} \quad, \quad \mathcal{P}^{\prime \prime \prime}(z)=12 \mathcal{P}(z) \mathcal{P}^{\prime}(z) .
$$

And by induction we conclude that $\mathcal{P}^{(2 n-2)}(z)$ and $\mathcal{P}^{(2 n+1)}(z) / \mathcal{P}^{\prime}(z)$ are polynomials of degree $n$ in $\mathcal{P}(z)$.

We can now describe the cut sphere representation of the torus. We consider the mapping

$$
\varsigma=\mathcal{P}(z)
$$

from the torus to the Riemann sphere (parameterized by $\varsigma$ ). Under this holomorphic mapping, every point of the sphere is reached exactly twice (counting multiplicity). The points which are reached once but have multiplicity two are those at which $\mathcal{P}^{\prime}$ is zero, these are $\varsigma_{1}, \varsigma_{2}$ and $\varsigma_{3}$. And $\varsigma_{4} \equiv \infty$ also has multiplicity two, as can be seen from (A.5). We can therefore make the mapping (A.12) one-to-one by considering, instead of the sphere, the Riemann surface obtained by taking two copies of the Riemann sphere, making cuts on each of these two sheets between $\varsigma_{1}$ and $\varsigma_{2}$ and between $\varsigma_{3}$ and $\infty$, and then gluing the two sheets together in such a way that when we go through a cut, we actually just go from one sheet to another (see figure 2). We will call this surface the cut sphere. It was constructed so as to make the holomorphic mapping (A.12) onto and one-to-one.

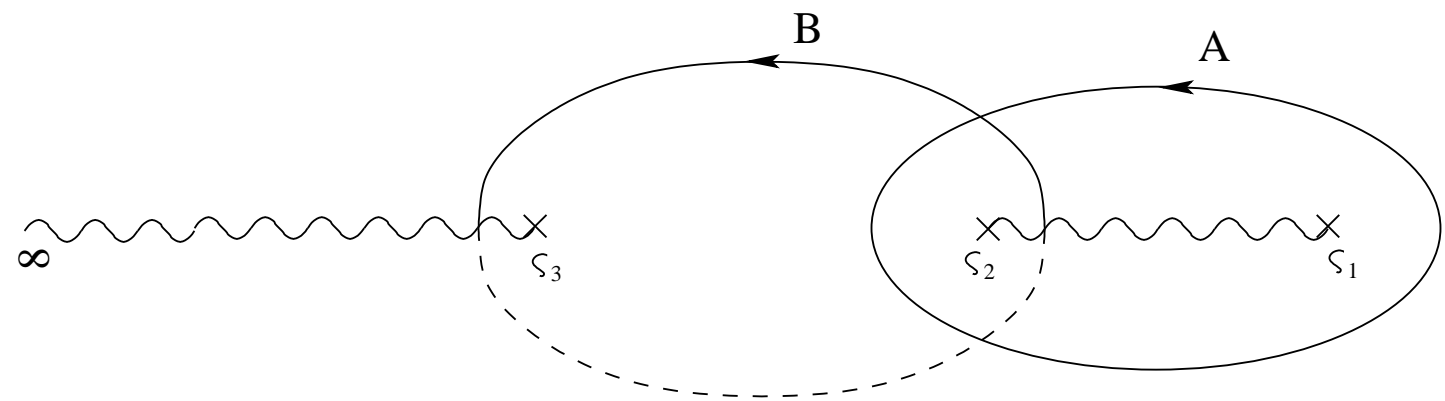

Figure 2: The cut sphere. The two cuts, drawn as wavy lines, are between $\varsigma_{1}$ and $\varsigma_{2}$ and between $\varsigma_{3}$ and $\infty$. We have drawn two independent nontrivial closed curves. The curve $A$ doesn't cross any cut, so it remains on the top sheet. The curve $B$ however, crosses the cut between $\varsigma_{3}$ and $\infty$, where it goes to the lower sheet (it is then drawn with a dashed line). It then crosses the other cut and comes back to the top sheet.

We now want to find good local coordinates on the cut sphere. First, at a regular point $\varsigma_{0}$ where $\mathcal{P}^{\prime}(z) \neq 0$, we have

$$
\varsigma=\varsigma_{0}+a_{1} z+a_{2} z^{2}+\ldots \quad a_{1} \neq 0
$$

and therefore $\varsigma-\varsigma_{0}$ is a good local coordinate. Now, at the points $\varsigma_{i}$, where $\mathcal{P}^{\prime}(z)=0$, we have

$$
\varsigma=\varsigma_{i}+0 \cdot z+a_{2} z^{2}+\ldots \quad a_{2} \neq 0
$$


therefore

$$
z \sim \frac{1}{\sqrt{a_{2}}} \sqrt{\varsigma-\varsigma_{i}}
$$

hence a good local coordinate is

$$
\varrho_{i}=\sqrt{\varsigma-\varsigma_{i}} .
$$

Now we would like to find the reciprocal of (A.12). For this we start by defining

$$
Q(\varsigma)=-2 \sqrt{\left(\varsigma-\varsigma_{1}\right)\left(\varsigma-\varsigma_{2}\right)\left(\varsigma-\varsigma_{3}\right)}
$$

Let us consider the integral

$$
I=\int_{\infty}^{\mathcal{P}(z)} \frac{\varsigma^{\prime}}{Q\left(\varsigma^{\prime}\right)},
$$

and let us make the change of variable $\varsigma^{\prime}=\mathcal{P}\left(z^{\prime}\right)$, then we see from (A.10) that $Q\left(\varsigma^{\prime}\right)=$ $\mathcal{P}^{\prime}\left(z^{\prime}\right)$. Therefore

$$
I=\int_{0}^{z} d z^{\prime}=z
$$

and we thus find the inversion formula

$$
z=\int_{\infty}^{\varsigma} \frac{d \varsigma^{\prime}}{Q\left(\varsigma^{\prime}\right)},
$$

where $\varsigma=\mathcal{P}(z)$. Note that we haven't defined the path of integration. And since there are nontrivial closed paths going around the cuts (like the path $A$ of Figure 2) or going through both cuts (like the path $B$ ), it seems that which path we choose will matter. What happens actually is that by going around a closed path, the integral picks up an integer combination of the periods

$$
\oint \frac{d \varsigma^{\prime}}{Q\left(\varsigma^{\prime}\right)}=2 m w+2 n w^{\prime}
$$

and therefore $z$ is unaffected due to the identifications (A.1). We can actually be more precise, looking back at Figure 2 and the contours $A$ and $B$, we can see that

$$
\oint_{A} \frac{d \varsigma^{\prime}}{Q\left(\varsigma^{\prime}\right)}=2 w^{\prime} \quad, \quad \oint_{B} \frac{d \varsigma^{\prime}}{Q\left(\varsigma^{\prime}\right)}=-2 w
$$

Indeed, in the $z$ plane, the contours $A$ and $B$ go respectively from $w$ to $w+2 w^{\prime}$ and from $2 w+w^{\prime}$ to $w^{\prime}$, winding only once on the torus. The result then follows from (A.18).

We now consider other useful functions, which we are using in this paper. First, the zeta function is minus the integral of the $\mathcal{P}$ function

$$
\mathcal{P}(z)=-\zeta^{\prime}(z)
$$


This defines $\zeta(z)$ up to a constant, which we choose so that the series expansion starts like

$$
\zeta(z)=\frac{1}{z}-\frac{g_{2}}{60} z^{3}-\frac{g_{3}}{140} z^{5}-\ldots
$$

The zeta function is not doubly periodic, but as can be seen from (A.21), under translation by a period it changes only by a constant (independent of $z$ )

$$
\zeta(z+2 w)=\zeta(z)+2 \eta \quad, \quad \zeta\left(z+2 w^{\prime}\right)=\zeta(z)+2 \eta^{\prime} .
$$

The two constants $\eta$ and $\eta^{\prime}$ are the values of the zeta functions at the half periods:

$$
\eta=\zeta(w) \quad, \quad \eta^{\prime}=\zeta\left(w^{\prime}\right) .
$$

By integrating $\zeta(z)$ around a fundamental parallelogram, one finds the Legendre's relation

$$
\eta w^{\prime}-\eta^{\prime} w=\frac{i \pi}{2}
$$

Another useful function is the $\sigma$ function which is defined by

$$
\zeta(z)=\frac{d}{d z} \ln \sigma(z)
$$

and normalized such that

$$
\sigma(z)=z-\frac{g_{2}}{240} z^{5}-\frac{g_{3}}{840} z^{7}-\ldots
$$

Again, this is not a doubly periodic function; under a shift by a period, it transforms as

$$
\sigma(z+2 w)=-\sigma(z) e^{2 \eta(z+w)} \quad, \quad \sigma\left(z+2 w^{\prime}\right)=-\sigma(z) e^{2 \eta^{\prime}\left(z+w^{\prime}\right)} .
$$

It is useful to relate the previous functions to the four Jacobi theta functions. We recall here their definitions as infinite sums

$$
\begin{gathered}
\theta_{1}(v)=\theta_{1}(v \mid \tau)=i \sum_{n=-\infty}^{\infty}(-1)^{n} q^{\left(n-\frac{1}{2}\right)^{2}} e^{i \pi(2 n-1) v} \\
\theta_{2}(v)=\theta_{2}(v \mid \tau)=\sum_{n=-\infty}^{\infty} q^{\left(n-\frac{1}{2}\right)^{2}} e^{i \pi(2 n-1) v} \\
\theta_{3}(v)=\theta_{3}(v \mid \tau)=\sum_{n=-\infty}^{\infty} q^{n^{2}} e^{i \pi 2 n v}
\end{gathered}
$$




$$
\theta_{4}(v)=\theta_{4}(v \mid \tau)=\sum_{n=-\infty}^{\infty}(-1)^{n} q^{n^{2}} e^{i \pi 2 n v}
$$

where $q=e^{i \pi \tau}=e^{i \pi \frac{w^{\prime}}{w}}$. They can also be expressed as infinite products

$$
\begin{gathered}
\theta_{1}(v)=2 f_{0}\left(q^{2}\right) q^{\frac{1}{4}} \sin \pi v \prod_{n=1}^{\infty}\left(1-2 q^{2 n} \cos 2 \pi v+q^{4 n}\right) \\
\theta_{2}(v)=2 f_{0}\left(q^{2}\right) q^{\frac{1}{4}} \cos \pi v \prod_{n=1}^{\infty}\left(1+2 q^{2 n} \cos 2 \pi v+q^{4 n}\right) \\
\theta_{3}(v)=f_{0}\left(q^{2}\right) \prod_{n=1}^{\infty}\left(1+2 q^{2 n-1} \cos 2 \pi v+q^{4 n-2}\right) \\
\theta_{4}(v)=f_{0}\left(q^{2}\right) \prod_{n=1}^{\infty}\left(1-2 q^{2 n-1} \cos 2 \pi v+q^{4 n-2}\right)
\end{gathered}
$$

where we have defined

$$
f_{0}\left(q^{2}\right)=\prod_{n=1}^{\infty}\left(1-q^{2 n}\right) .
$$

In all these and following expressions, $v$ is related to $z$ by

$$
v=\frac{z}{2 w}
$$

In other words, the periods in the $v$ plane are 1 and $\tau$. The theta functions obey the following periodicity equations.

$$
\begin{gathered}
\theta_{1}(v+1)=-\theta_{1}(v) \quad, \quad \theta_{1}(v+\tau)=-e^{-i \pi(2 v+\tau)} \theta_{1}(v) \\
\theta_{2}(v+1)=-\theta_{2}(v), \quad \theta_{2}(v+\tau)=e^{-i \pi(2 v+\tau)} \theta_{2}(v) \\
\theta_{3}(v+1)=\theta_{3}(v), \quad \theta_{3}(v+\tau)=e^{-i \pi(2 v+\tau)} \theta_{3}(v) \\
\theta_{4}(v+1)=\theta_{4}(v), \quad \theta_{4}(v+\tau)=-e^{-i \pi(2 v+\tau)} \theta_{4}(v)
\end{gathered}
$$

The theta functions are related to each other by translations by half periods. For example for $\theta_{1}$ we have

$$
\theta_{1}\left(v+\frac{1}{2}\right)=\theta_{2}(v) \quad, \quad \theta_{1}\left(v+\frac{\tau}{2}\right)=i e^{-i \pi\left(v+\frac{\tau}{4}\right)} \theta_{4}(v)
$$

and we have similar relations for the other theta functions. We can express all the functions defined above from the Weierstrass functions, in terms of theta functions, For example we have

$$
\zeta(z)=\frac{\eta}{w} z+\frac{1}{2 w} \frac{\theta_{1}^{\prime}(v)}{\theta_{1}(v)}
$$




$$
\sigma(z)=2 w \exp \left(\frac{\eta z^{2}}{2 w}\right) \frac{\theta_{1}(v)}{\theta_{1}^{\prime}(0)}
$$

and

$$
\eta=-\frac{1}{12 w} \frac{\theta_{1}^{\prime \prime \prime}(0)}{\theta_{1}^{\prime}(0)} \quad, \quad \eta^{\prime}=-\frac{i \pi}{2 w}-\frac{\tau}{12 w} \frac{\theta_{1}^{\prime \prime \prime}(0)}{\theta_{1}^{\prime}(0)} .
$$

The theta functions obey many more relations; here we have only written the ones that we are using in this paper. For a more detailed introduction, see for example [24].

\section{References}

[1] V. Alessandrini, D. Amati and B. Morel, The Asymptotic Behaviour of the Dual Pomeron Amplitude, Il Nuovo Cimento, vol7 (1972) 797.

[2] D. J. Gross and P. F. Mende, The High-Energy Behavior Of String Scattering Amplitudes, Phys. Lett. B 197 (1987) 129.

[3] D. J. Gross and P. F. Mende, String Theory Beyond The Planck Scale, Nucl. Phys. B 303 (1988) 407.

[4] D. J. Gross and J. L. Manes, The High-Energy Behavior Of Open String Scattering, Nucl. Phys. B 326 (1989) 73.

[5] D. J. Gross, High-Energy Symmetries Of String Theory, Phys. Rev. Lett. 60 (1988) 1229.

[6] C. T. Chan and J. C. Lee, Stringy symmetries and their high-energy limits, Phys. Lett. B 611 (2005) 193 arXiv:hep-th/0312226; C. T. Chan and J. C. Lee, Zeronorm states and high-energy symmetries of string theory, Nucl. Phys. B 690 (2004) 3 arXiv:hep-th/0401133; C. T. Chan, P. M. Ho and J. C. Lee, Ward identities and highenergy scattering amplitudes in string theory, Nucl. Phys. B 708 (2005) 99 arXiv:hepth/0410194]; C. T. Chan, P. M. Ho, J. C. Lee, S. Teraguchi and Y. Yang, Solving all 4-point correlation functions for bosonic open string theory in the high energy limit, arXiv:hep-th/0504138; C. T. Chan, P. M. Ho, J. C. Lee, S. Teraguchi and Y. Yang, High-energy zero-norm states and symmetries of string theory, arXiv:hep-th/0505035.

[7] P. West, $E_{11}$ and M Theory, Class.Quant.Grav. 18 (2001) 4443-4460, hep-th/0104081.

[8] V. Alessandrini, D. Amati, M. Le Bellac and D. Olive, The Operator Approach to Dual Multiparticle Theory, Phys. Reports 6 (1971) 269; D. Ebert and H. Otto, A survey on Dual Tree and Loop Amplitudes, Zeitschrift Fortschritte der Physik, 25 (1977) 203.

[9] A. Neveu and P. C. West, Conformal Mappings and the Three String Bosonic Vertex; Phys. Lett. B179 235 (1986).

[10] A. Neveu and P. C. West, The Cyclic Symmetric Vertex for Three Arbitrary NeveuSchwartz Strings; Phys. Lett. B180 34 (1986).

[11] A. Neveu and P. C. West, Group Theoretic Approach To The Perturbative String S Matrix, Phys. Lett. B 193 (1987) 187.

[12] A. Neveu and P. C. West, A Group Theoretic Method For String Loop Diagram, Phys. Lett. B 194 (1987) 200.

[13] A. Neveu and P. C. West, Group Theoretic Approach To The Open Bosonic String Multiloop S Matrix, Commun. Math. Phys. 114 (1988) 613.

[14] A. Neveu and P. C. West, Cycling, Twisting And Sewing In The Group Theoretic Approach To Strings, Commun. Math. Phys. 119 (1988) 585. 
[15] P. West and M. Freeman, Ghost Vertices for the Bosonic String using the Group Theoretical Approach to String Theory; Phys. Lett. B205 30 (1988).

[16] P. West, Multiloop Ghost Vertices and the Determination of the Multiloop Measure; Phys. Lett. B205 38 (1988).

[17] P. West, String Vertices and Induced Representations; Nucl. Phys. B320 103 (1989).

[18] P. C. West, A Brief Review Of The Group Theoretic Approach To String Theory, A Brief Review of the Group Theoretic Approach to String Theory, in "Conformal Field Theories and Related Topics", Proceedings of Third Annecy Meeting on Theoretical Physics, LAPP, Annecy le Vieux, France, Nucl. Phys. B (Proc. Suppl) 5B (1988) 217, edited by P. Binütruy, P. Sorba and R. Stora, North Holland (1988); and "Ninth Workshop on Grand Unification"; edited by R. Barloutaud, World Scientific (1988); and "1988 Electroweak Interactions and Unified Theories", Proceedings of the XXIIIrd Rencontre de Moriond, edited by J. Tran Thanh Van, published by Edition Frontiéres (1988). CERN-TH-5059-88

[19] A. Neveu and P. C. West, Neveu-Schwarz Excited String Scattering: A Superconformal Group Computation, Phys. Lett. B 200 (1988) 275; A. Neveu and P. C. West, Group Theoretic Approach To The Superstring And Its Supermoduli, Nucl. Phys. B 311 (1988) 79.

[20] M. D. Freeman and P. C. West, Ramond String Scattering In The Group Theoretic Approach To String Theory, Phys. Lett. B 217 (1989) 259.

[21] A. Neveu and P. C. West, Symmetries of the Interacting Gauge Covariant Bosonic String; Nucl. Phys. B278 601 (1986).

[22] R. W. Gebert, H. Nicolai and P. C. West, Multistring vertices and hyperbolic KacMoody algebras, Int. J. Mod. Phys. A 11 (1996) 429 arXiv:hep-th/9505106.

[23] L. Alvarez-Gaume, C. Gomez, G. Moore and C. Vafa, Strings in the Operator Formalism, Nucl. Phys. B303 (1988) 455.

[24] A. Erdelyi, Bateman Manuscript Project vol II, McGraw-Hill, (1953).

[25] L. Alvarez-Gaume, C. Gomez and C. Reina, Loop Groups, Grassmanians and String Theory, Phys. Lett. B 190 (1987) 55; C. Vafa, Operator Formulation on Riemann Surfaces, Phys. Lett. B 190 (1987) 47.

[26] D. Amati, C. Bouchiat, J. Gervais, On the building of dual diagrams from unitarity, Lett. Nuovo cimento 2 (1969) 399; K. Bardacki, M. Halpern and J. Shapiro, Unitary Closed Loops in Reggeized Feynman theory, Phys. Rev. D185 (1969) 1910.

[27] D. Gross, A. Neveu, J. Scherk and J. Schwarz, Renormalisation and unitarity in the dual-resonance model, Phys. Rev. D2 (1970) 697.

[28] A. Neveu and J. Scherk, Parameter-free regularization of one loop unitary diagram, Phys. Rev. D1 (1970) 311.

[29] D. Gross, and J. Schwarz, Basic operators of the dual resonance model, Nucl. Phys, B23 (1970) 333.

[30] V. Alessandrini, A General Approach To Dual Multiloop Diagrams, Nuovo Cim. A 2 (1971) 321.

[31] V. Alessandrini and D. Amati, Properties Of Dual Multiloop Amplitudes, Nuovo Cim. A 4 (1971) 793.

[32] E. Cremmer, Nucl. Phys, B31 (1971) 477. 
[33] L. Brink and D. Olive, Nucl. Phys, B58 (1973) 237; L. Brink and D. Olive, Nucl. Phys, B56 (1973) 253.

[34] O. Alvarez, Theory Of Strings With Boundaries: Fluctuations, Topology, And Quantum Geometry, Nucl. Phys. B 216 (1983) 125; E. D'Hoker and D. H. Phong, Multiloop Amplitudes For The Bosonic Polyakov String, Nucl. Phys. B 269 (1986) 205; G. W. Moore and P. Nelson, Absence Of Nonlocal Anomalies In The Polyakov String, Nucl. Phys. B 266 (1986) 58; J. Polchinski, Evaluation Of The One Loop String Path Integral, Commun. Math. Phys. 104 (1986) 37. 